\title{
Spectral and Scattering Theory for Symmetric Hyperbolic Systems in an Exterior Domain
}

\author{
By \\ Kiyoshi MochIzUKI*
}

\section{Introduction}

The purpose of this research is to treat by means of the perturbation method the spectral theory and the scattering theory connected with the exterior problem for symmetric hyperbolic systems.

The systems which we shall consider are of the form

$$
\frac{1}{i} M(x) \frac{\partial u}{\partial t}=L u=\frac{1}{i} \sum_{j=1}^{n} A_{j}(x) \frac{\partial u}{\partial x_{j}}+B(x) u
$$

for $x$ in an exterior domain $G$ of $\boldsymbol{R}^{n}(n \geq 2)$ and $t \in \boldsymbol{R}^{1}$ (time), where $i=\sqrt{-1}, u=u(x, t)$ is a vector valued function whose values lie in the $m$-dimensional complex space $\boldsymbol{C}^{m}$, and $M(x), A_{j}(x)$ and $B(x)$ are $m \times m$ matrix valued function such that, for each $x$ in $G, M(x)$ is positive definite, $A_{j}(x)$ are Hermitian and $\sum_{j=1}^{n} \frac{\partial A_{j}(x)}{\partial x_{j}}=i\left\{B(x)-B(x)^{*}\right\}$ $\left(B(x)^{*}\right.$ denotes the adjoint matrix of $\left.B(x)\right)$. The boundary $\partial G$ of $G$ is a compact hypersurface of class $C^{2}$ in $\boldsymbol{R}^{n}$. We put the boundary conditions in the following form: At each point $z$ of the boundary, let $N(z)$ denote a linear subspace of $\boldsymbol{C}^{m}$ of constant dimension which is smoothly varying on $\partial G$. Then we require for all time $t$ that

$$
u(z, t) \text { lies in } N(z) \text { at each point } z \text { of } \partial G \text {. }
$$

The boundary space $N(z)$ is assumed to be maximally conservative with respect to $L$ and coercive for $L$ restricted to the orthocomplement of its null space.

Received July 7, 1969.

Communicated by S. Matsuura.

* Yoshida College, Kyoto University. 
Throughout this paper, we shall restrict ourselves to perturbations taking place in bounded domains; i.e., we assume that, outside of a sufficiently large ball, say for $|x|>\rho, M(x)$ is the identity matrix, $A_{j}(x)$ do not depend on $x$ and $B(x)$ is zero. We write $A_{j}(x)=A_{j}$ (constant) for $|x|>\rho$. Then the unperturbed problem compared with (1.1), (1.2) is the initial value problem for the equation

$$
\frac{1}{i} \frac{\partial u^{0}}{\partial t}=L_{0} u^{0}=\frac{1}{i} \sum_{j=1}^{n} A_{j} \frac{\partial u^{0}}{\partial x_{j}} \quad\left(x \in \boldsymbol{R}^{n}, t \in \mathbb{R}^{1}\right) .
$$

The matrix $A^{0}(\xi)=\sum_{j=1}^{n} A_{j} \xi_{j}\left(\xi \in \boldsymbol{R}^{n}-\{0\}\right)$ is not assumed to be nonsingular. But it is assumed to be isotropic:

(1.4) $\operatorname{det}\left[A^{0}(\xi)-\lambda I\right]=\prod_{\nu=1}^{k}\left(\tau_{\nu}|\xi|-\lambda\right)^{m_{\nu}}, \sum_{\nu=1}^{k} m_{\nu}=m, \tau_{1}>\tau_{2}>\cdots>\tau_{k}$.

The scattering theory for symmetric hyperbolic systems in an exterior domain is already treated by Lax and Phillips in their book [12] as an application of their abstract representation theorems, based on their introduction and study of outgoing and incoming subspaces of initial data of the problem. However, their theory is limited to the case when the space dimension $n$ is odd and $L$ is elliptic; i. e., the matrix $A(x, \xi)=\sum_{j=1}^{n} A_{j}(x) \xi_{j}$ is non-singular for each $x \in G$ and $\xi \in \mathbb{R}^{n}-\{0\}$, though condition (1.4) on the unperturbed operator $L_{0}$ is not assumed there.

Condition (1.4) is very strong. However, there exist several concrete problems appearing in classical physics which satisfy this condition (cf., e.g., Wilcox [22]). The Maxwell equations are a typical example.

We require (1.4) mainly to give an explicit formulation of the radiation conditions (see Definition 4.1). Our formulation differs from that given by Lax and Phillips, and is based on the work [15] of Matsumura. ${ }^{1)}$ Once we succeed to define the radiation conditions attached to the operator $L$, all the results can be easily treated in the framework of the theory developed by many authors for the

1) In $[15,16]$ Matsmura proved the so-called decay principle for symmetric hyperbolic systems with constant coefficients in the half space $R_{+}^{n}$. 
wave and Schrödinger equations.

We denote by $\mathscr{H}_{1}$ the Hilbert space of $\boldsymbol{C}^{m}$-valued square integrable functions in $G$ with norm

$$
\|f\|_{1}=\left\{\int_{G} M(x) f(x) \cdot \overline{f(x)} d x\right\}^{1 / 2},
$$

where $\zeta \cdot \bar{\eta}$ denotes the inner product between $\zeta$ and $\eta$ in $\boldsymbol{C}^{m}$. By assumptions, the operator $L_{1}=M^{-1}(x) L$ initially defined for smooth functions with bounded supports in $\bar{G}$ which satisfy the boundary condition (1.2) determines uniquely a selfadjoint operator in $\mathscr{H}_{1}$, which we also denote by $L_{1}$. Our main problem is to prove the following assertion: Let $\sigma(\neq 0)$ real be not in the point spectrum of $L_{1}$, and let $v_{\varepsilon}$ be the solution in $\mathscr{H}_{1}$ of the problem

$$
L_{1} v_{\varepsilon}-(\sigma+i \varepsilon) v_{\varepsilon}=g(x) \quad(\varepsilon \neq 0, \text { real })
$$

with $g(x) \in \mathscr{H}_{1}$ having bounded support. Then, as $\varepsilon \rightarrow \pm 0$, the limits $v_{ \pm}$of $v_{\varepsilon}$ exist and satisfy, though not in $\mathscr{H}_{1}$, the equation

$$
M^{-1}(x) L v_{ \pm}-\sigma v_{ \pm}=g(x),
$$

the boundary condition (1.2) and the radiation conditions at infinity (the subscripts "+" and "-" are related with the incoming and outgoing radiation conditions, respectively). To prove this we can apply a method developed by Éidus [2] for a generalized Helmholtz equation, noting the local compactness of the set

$$
\left\{f \in \mathscr{D}\left(L_{1}\right) ; f \perp \Re\left(L_{1}\right),\|f\|_{1}+\left\|L_{1} f\right\|_{1} \leq 1\right\},
$$

which is deduced from the coercivity condition on $N(z)$.

The above assertion derives not only the absolute continuity of the continuous spectrum of $L_{1}$ but also the existence of the distorted plane waves $\Phi_{\nu}^{ \pm}(x, \xi)=P_{\nu}(\xi) e^{i x_{\bullet} \xi}+V_{\nu}^{ \pm}(x . \xi)$ solving the equation

$$
M^{-1}(x) L \Phi_{\nu}^{ \pm}(x, \xi)-\tau_{\nu}|\xi| \Phi_{\nu}^{ \pm}(x, \xi)=0 \quad(\nu \neq(k+1) / 2)
$$

with the boundary condition (1.2), where $P_{\nu}(\xi)$ are the projections in $\boldsymbol{C}^{m}$ onto the eigenspaces of the matrix $A^{0}(\xi)$ corresponding to the eigenvalues $\tau_{\nu}|\xi|$, and $V_{\nu}^{-}(x, \xi)$ and $V_{\nu}^{+}(x, \xi)$ are the scattered waves satisfying the incoming and outgoing radiation conditions, 
respectively. ${ }^{2)}$ Now we can construct the "wave operators" $W^{ \pm}$in the following form :

$$
\left[W^{ \pm} f\right](x)=(2 \pi)^{-n / 2} \sum_{\nu=1}^{[k / 2]} \int_{R^{n}}\left\{\Phi_{\nu}^{ \pm}(x, \xi)+\Phi_{k-\nu+1}^{ \pm}(x, \xi)\right\} \hat{f}(\xi) d \xi,
$$

where the integrals are taken in the sense of the limit in the mean, and $\hat{f}(\xi)$ denotes the Fourier transform of $f(x)$ in $\mathscr{H}_{0}=$ $\left[L^{2}\left(\boldsymbol{R}^{n}\right)\right]^{m}$ :

$$
\hat{f}(\xi)=(2 \pi)^{-n / 2} \int_{\boldsymbol{R}^{n}} e^{-i x \cdot \xi} f(x) d x=\sum_{\nu=1}^{k} P_{\nu}(\xi) \hat{f}(\xi) .
$$

We denote by $Q_{0}$ and $Q$ the projections onto the null space of $L_{0}$ and the eigenspace of $L_{1}$, respectively. Then $W^{ \pm}$define unitary operators of $\left(I-Q_{0}\right) \mathscr{H}_{0}$ onto $(I-Q) \mathcal{H}_{1}$, and coincide with the wave operators in the time-dependent formulation, namely

$$
W^{ \pm}=s-\lim _{t \rightarrow \pm \infty} e^{i L_{1} t} J e^{-i L_{0} t}\left(I-Q_{0}\right)
$$

where $J: \mathscr{H}_{0} \rightarrow \mathscr{H}_{1}$ is the "trücation" operator defined by

$$
[J f](x)=f(x) \quad(x \in G) .
$$

As is mentioned above, our results cover the scattering problem for the Maxwell equations of the form :

$$
\left[\begin{array}{cc}
M_{1}(x) & 0 \\
0 & M_{2}(x)
\end{array}\right] \frac{\partial}{\partial t}\left[\begin{array}{l}
u_{1} \\
u_{2}
\end{array}\right]=\left[\begin{array}{cc}
0 & \operatorname{curl} \\
-\operatorname{curl} & 0
\end{array}\right]\left[\begin{array}{l}
u_{1} \\
u_{2}
\end{array}\right]
$$

with the boundary of a perfect conductor. This generalizes results of Schmidt [19], in which is developed the case where $M_{1}(x)=M_{2}(x)$ $\equiv I$. Our results also have some contact with the work of Wilcox [22]. He considered uniformly propagative systems of the form

$$
M(x) \frac{\partial u}{\partial t}=\sum_{j=1}^{n} A_{j} \frac{\partial u}{\partial x_{j}} \quad\left(A_{j}: \text { constant }\right)
$$

in the whole space $\boldsymbol{R}^{n}$. Here the operator $\sum_{j=1}^{n} A_{j} \frac{\partial u}{\partial x_{j}}$ is called to be uniformly propagative if

2) Note that the incoming wave $V_{\bar{\nu}}(x, \xi)$ is the incoming solution $v_{+}(x, \sigma)$ of an equation of type (1.7), and similarly for $V_{\nu}^{+}(x, \xi)$. 


$$
\operatorname{det}\left[\sum_{j=1}^{n} A_{j} \xi_{j}-\lambda I\right]=\prod_{\nu=1}^{k}\left(\tau_{\nu}(\xi)-\lambda\right)^{m_{\nu}}, \sum_{\nu=1}^{k} m_{\nu}=m,
$$

where $\tau_{\nu}(\xi) \neq 0$ for any $\xi \in \boldsymbol{R}^{n}-\{0\}$ or $\tau_{\nu}(\xi) \equiv 0$. He assumed that

$$
M(x)-I=o\left(|x|^{-1}\right) \quad \text { as } \quad|x| \rightarrow \infty
$$

and derived the existence of the wave operators $W^{ \pm 3}$. However, in his paper, it remains as an open problem whether $W^{ \pm}$are unitary. Our results can be applied to solve this problem for a restricted case (see (1.4)).

Let us explain briefly the outline of our proof. In $\S 2$ we summarize several assumptions required below, and give a Hilbert space formulation of the problem. In $\S 3$ we consider the unperturbed problem. We summarize and slightly sharpen results of Matsumura [15] concerning the elementary solution of $L_{0}-\lambda I$. All the discussions in $\S 3$ are used to study the structure of the spectrum of $L_{1}$. In $\$ 4$, after giving a formulation of the radiation conditions, we derive that the point spectrum $\sigma_{p}\left(L_{1}\right)$ of $L_{1}$ consists of isolated eigenvalue which are of finite multiplicity except the origin 0 , and that the continuous spectrum is absolutely continuous with respect to the Lebesgue measure showing that the solution of (1.6) with $\sigma$ not in the set $\sigma_{p}\left(L_{1}\right) \cup\{0\}$ converges as $\varepsilon \rightarrow \pm 0$ in the class of functions which satisfy the radiation conditions. In $\S 5$ we establish the existence and certain properties of the distorted plane waves $\Phi_{\nu}^{ \pm}(x, \xi)$, which provide the eigenfunction expansions associated with the operator $L_{1}$. The operators $W^{ \pm}$defined by (1.9) are shown to be unitary operators from $\left(I-Q_{0}\right) \mathscr{H}_{0}$ onto $(I-Q) \mathcal{H}_{1}$ :

$$
I-Q=W^{ \pm} Z^{ \pm} \text {and } I-Q_{0}=Z^{ \pm} W^{ \pm},
$$

where $Z^{ \pm}$are the adjoint operators of $W^{ \pm}$given for each $f(x)$ $\in(I-Q) \mathcal{H}_{1}$ by

$$
(1.18)\left[Z^{ \pm} f\right]^{\wedge}(\xi)=(2 \pi)^{-n / 2} \sum_{\nu=1}^{[k / 2]} \int_{G}\left\{\Phi_{\nu}^{ \pm *}(x, \xi)+\Phi_{k-\nu \mid 1}^{ \pm *}(x, \xi)\right\} M(x) f(x) d x
$$

The first relation of (1.17) gives the expansion formula (generalized Fourier inversion formula) for $L_{1}$ restricted to $(I-Q) \mathcal{H}_{1}$. Further,

3) Similar problems have been treated also by Kato [10] and Ikebe [7]. 
(1.17) establishes the unitary equivalence between $L_{0}$ and $L_{1}$ :

$$
L_{1}(I-Q)=W^{ \pm} L_{0}\left(I-Q_{0}\right) Z^{ \pm} \quad\left(Z^{ \pm}=W^{ \pm-1}\right) .
$$

In $\S 6$ we use (1.19) to show that $W^{ \pm}$defined by (1.9) coincide with the wave operators in the time dependent formulations. Then the scattering operator $S$ can be defined as $S=Z^{+} W^{-}$, and is proved to be unitary in $\left(I-Q_{0}\right) \mathscr{H}_{0}$. Finally, in $\S 7$ we apply the obtained results to the Maxwell equations (1.13) in an exterior domain and to the uniformly propagative systems (1.14) in the whole space $\boldsymbol{R}^{n}$.

\section{Assumptions and the Hilbert Space Formulation of the Problem}

We consider the solution with finite energy (i.e., square integrable in the exterior domain $G$ of $\boldsymbol{R}^{n}$ ) of the mixed initial-boundary value problem

(2.1) $\frac{1}{i} M(x) \frac{\partial u}{\partial t}=L u=\frac{1}{i} \sum_{j=1}^{n} A_{j}(x) \frac{\partial u}{\partial x_{j}}+B(x) u \quad$ for $\quad x \in G, t \in \boldsymbol{R}^{1}$;

$$
\begin{array}{ll}
u(x, 0)=u_{0}(x) & \text { for } \quad x \in G ; \\
u(z, t) \in N(z) & \text { for } \quad z \in \partial G, t \in \boldsymbol{R}^{1} .
\end{array}
$$

In the following, we require on the coefficients of (2.1) and the boundary space $N(z)$ the following assumptions.

(i ) $M(x)$ is Hermitian, positive definite, and bounded measurable in $x \in G ; A_{j}(x)$ are Hermitian, and continuously differentiable in $x ; B(x)$ is continuous in $x$.

(ii) The differential operator $L$ is formally selfadjoint:

$$
\sum_{j=1}^{n} \frac{\partial A_{j}(x)}{\partial x_{j}}=i\left\{B(x)-B^{*}(x)\right\} \quad \text { for each } x \text { in } G .
$$

(iii) Outside of a sufficiently large ball, say for $|x|>\rho$,

$$
M(x)=I, A_{j}(x)=A_{j} \text { (constant) and } B(x)=0 .
$$

(iv) We set $A(x, \xi)=\sum_{j=1}^{n} A_{j}(x) \xi_{j}$ and $A^{0}(\xi)=\sum_{j=1}^{n} A_{j} \xi_{j}\left(\xi \in \mathbb{R}^{n}\right)$. Then we require that $A(z, n(z)), n(z)$ denoting the outer normal to $\partial G$ at $z$, is of constant rank near the boundary, and that $A^{0}(\xi)$ is isotropic: 


$$
\operatorname{det}\left[A^{0}(\xi)-\lambda I\right]=\prod_{\nu=1}^{k}\left(\tau_{\nu}|\xi|-\lambda\right)^{m_{\nu}}, \sum_{\nu=1}^{k} m_{\nu}=m \text {, }
$$

for each $\xi \in \boldsymbol{R}^{n}$ and $\lambda \in \boldsymbol{C}^{1}$, where we label $\left\{\tau_{\nu}\right\}$ in decreasing order:

$$
\tau_{1}>\tau_{2}>\cdots>\tau_{k}
$$

(v) The boundary $\partial G$ is contained in the ball $\{|x|<\rho\}$. The boundary space $N(z)$ is maximally conservative with respect to $L$ :

$$
A(z, n(z)) \zeta \cdot \bar{\zeta}=0 \quad \text { for each } \zeta \text { in } N(z) \text {, }
$$

and $N(z)$ is not properly contained in any other subspace of $\boldsymbol{C}^{m}$ having this property.

Remark 2.1. A necessary and sufficient condition for $N(z)$ being maximally conservative is given by Lax and Phillips [12] (Lemma 2.1 of Chapter VI). They considered the case where $A(z, n(z))$ is non-singular at each point $z$ of $\partial G$. However, their results are applicable also in our case since we assumed that $A(z, n(z))$ is of constant rank near the boundary.

Under these assumptions, it is known (see Lax and Phillips [11], $\S 3)$ that the differential operator $L$ is essentially selfadjoint in the Hilbert space $\mathscr{H}=\left[L^{2}(G)\right]^{m}$ with norm

$$
\|f\|=\left\{\int_{G}|f(x)|^{2} d x\right\}^{1 / 2}
$$

as defined for differentiable functions in $\mathscr{H}$ which satisfy the boundary condition (2.3). ${ }^{4}$ We also denote by $L$ the selfadjoint extension. Then the domain of $L$ is given by

(2. 5) $\mathscr{D}(L)=\{f \in \mathscr{H} ; L f \in \mathcal{H}, f(z) \in N(z)$ in the strong sense $\}$,

where the derivative is considered in the distribution sense.

By assumptions (i), (iii) on $M(x)$, we see that there exists a positive constant $C$ such that

$$
C^{-2}|\zeta|^{2} \leq M(x) \zeta \cdot \bar{\zeta} \leq C^{2}|\zeta|^{2} \quad \text { for each } \zeta \text { in } C^{m}
$$

Thus, if we put

4) The Hilbert space formulation of the boundary value problem for symmetric first order differential equations was initiated by Friedrichs [4]. 


$$
\|f\|_{1}=\left\{\int_{G} M(x) f(x) \cdot \overline{f(x)} d x\right\}^{1 / 2},
$$

then it determines a new norm of $\left[L^{2}(G)\right]^{m}$ which is equivalent to (2.4). We denote by $\mathscr{H}_{1}$ the new Hilbert space with the norm defined by (2.7). Define the operator $L_{1}$ in $\mathscr{H}_{1}$ by

$$
L_{1}=M^{-1} L, \quad \mathscr{D}\left(L_{1}\right)=\mathscr{D}(L),
$$

where $M$ is the multiplication operator given by $M(x)$. Then obviously $L_{1}$ is a selfadjoint operator in $\mathscr{H}_{1}$. Thus, the problem (2.1), (2.2), (2.3) can be written in the following form:

$$
\frac{1}{i} \frac{\partial u}{\partial t}=L_{1} u,\left.\quad u\right|_{t=0}=u_{0} \in \mathscr{H}_{1} .
$$

In order to study the scattering operator for the group $\left\{e^{i L_{1} t}\right\}$ with respect to the unperturbed group $\left\{e^{i L_{0} t}\right\}$ we further require the following assumptions :

(vi) There exists a postitive constant $\widetilde{C}=\widetilde{C}\left(r^{\prime}, r\right)$ such that

$$
\sum_{j=1}^{n}\left\|\frac{\partial f}{\partial x_{j}}\right\|_{G_{r}} \leq \widetilde{C}\left\{\|f\|_{G_{r}}+\|L f\|_{G_{r}}\right\} \quad \text { (for any } \rho<r^{\prime}<r \text { ) }
$$

for all functions $f$ in $\mathscr{D}(L) \ominus \Re(L)$, where $G_{r}=\{x \in G ;|x|<r\}$, $\|f\|_{G_{r}}=\left\{\int_{G_{r}}|f(x)|^{2} d x\right\}^{1 / 2}$ and $\mathscr{N}(L)$ denotes the null space of $L$.

(vii) $M(x)$ is continuously differentiable in $x$, and $M L-L M$ defines a bounded operator in $\mathscr{H}$.

Remark 2.2. (vi) is a kind of coercivity condition on the boundary space $N(z)$. In the case when $L$ is elliptic, $N(z)$ is called coercive in the sense of Aronszajn if the inequality in (vi) holds for all $f$ in $\mathscr{D}(L)$ ([12]; VI, Definition 2.1 and Lemma 2.2). If $L$ is elliptic and $N(z)$ is coercive in the sense of Aronszajn, then assumption (vii) is not necessary in all the following discussions. In our assumption (vi), however, there exists a difficulty that the space $\mathscr{D}\left(L_{1}\right) \ominus$ $\Re\left(L_{1}\right)$ is not equal to $\mathscr{D}(L) \ominus \Re(L)$ unless $M(x) \equiv I$, though $\Re\left(L_{1}\right)$ $=\mathscr{N}(L)$. (vii) is used only to remove this difficulty.

Lemma 2.1. Under assumptions (vi) and (vii), there exists a positive constant $C_{1}=C\left(r^{\prime}, r\right)$ such that 
(2.10) $\sum_{j=1}^{n}\left\|\frac{\partial f}{\partial x_{j}}\right\|_{1, G_{r}} \leq C_{1}\left\{\|f\|_{1, G_{r}}+\left\|L_{1} f\right\|_{1_{0} G_{r}}\right\} \quad$ (for any $\left.\rho<r^{\prime}<r\right)$ for all functions $f$ in $\mathscr{D}\left(L_{1}\right) \ominus \mathscr{M}\left(L_{1}\right)$, where $\|f\|_{1, G r}$ $=\left\{\int_{G_{r}} M(x) f \cdot \bar{f} d x\right\}^{1 / 2}$.

Proof. Note that $f \in \mathscr{D}\left(L_{1}\right) \ominus \mathscr{M}\left(L_{1}\right)$ if and only if $M f \in \mathscr{D}(L) \ominus$ $\Re(L)$. Since $M(x)$ and $\partial M(x) / \partial x_{j}$ are all bounded in $x$, it follows that

$$
\begin{aligned}
\sum_{j=1}^{n}\left\|\frac{\partial f}{\partial x_{j}}\right\|_{G_{r}} & \leq \text { const }\left\{\sum_{j=1}^{n}\left\|\frac{\partial M(x) f}{\partial x_{j}}\right\|_{G_{r}}+\|f\|_{G_{r^{\prime}}}\right\} \\
& \leq \text { const }\left\{\tilde{C}\left\|M f !_{G_{r}}+\widetilde{C}\right\| L M f\left\|_{G_{r}}+\right\| f \|_{G_{r}}\right\}
\end{aligned}
$$

for $f \in \mathscr{D}\left(L_{1}\right) \ominus \Re\left(L_{1}\right)$. On the other hand, we have

$$
\|L M f\|_{G_{r}} \leq\|M L f\|_{G_{r}}+\|\{L M-M L\} f\|_{G_{r}},
$$

where $\{L M-M L\} f=0$ for $|x|>\rho$ by (iii). These prove the inequality of the lemma if we note that $M(x)$ has the bounded inverse $M^{-1}(x)$.

q. e. d.

\section{The Free Space Problem}

\section{1. Motivation.}

We consider the reduced equation

$$
L_{0} v^{0}-\lambda v^{0}=\frac{1}{i} \sum_{j=1}^{n} A_{j} \frac{\partial v^{0}}{\partial x_{j}}-\lambda v^{0}=f(x) \quad\left(\lambda \in C^{1}\right)
$$

to the unperturbed problem (1.3) in the whole space $\boldsymbol{R}^{n}$, where $A_{j}$ are $m \times m$ Hermitian matrices and $A^{0}(\xi)=\sum A_{j} \xi_{j}, \xi \in \boldsymbol{R}^{n}$, is assumed to be isotropic (see assumption (iv)).

We denote by $\mathscr{H}_{0}=\left[L^{2}\left(\boldsymbol{R}^{n}\right)\right]^{m}$ the Hilbert space with norm

$$
\|f\|_{0}=\left\{\int_{\boldsymbol{R}^{n}} f(x) \cdot \overline{f(x)} d x\right\}^{1 / 2} .
$$

Then, obviously $L_{0}$ defines a selfadjoint operator in $\mathscr{H}_{0}$, which we also denote by $L_{0}$, with domain

$$
\mathscr{D}\left(L_{0}\right)=\left\{f ; \hat{f}(\xi), A^{\mathrm{n}}(\xi) \hat{f}(\xi) \in \hat{\mathcal{H}}_{0}\right\},
$$

where $\hat{f}(\xi)$ is the Fourier transform of $f(x)$ : 


$$
\hat{f}(\xi)=(2 \pi)^{-n / 2} \int_{R^{n}} f(x) e^{-i x \cdot \xi} d x=[\mathscr{L} f](\xi) .
$$

Since $A^{0}(\xi)$ is an odd function of $\xi$, it follows in (iv) that

$$
\tau_{\nu}=-\tau_{k-\nu+1} \text { and } m_{\nu}=m_{k-\nu+1} .
$$

Thus $\tau_{\nu} \neq 0$ for each $\nu$ if $k$ is even, and $\tau_{(k+1) / 2}=0$ if $k$ is odd. We denote by $P_{v}(\xi)(\nu=1,2, \cdots, k)$ the projection in $\boldsymbol{C}^{m}$ onto the eigenspace of $A^{0}(\xi)$ corresponding to the eigenvalue $\tau_{\nu}|\xi| . P_{\nu}(\xi)$ are Hermitian matrices obtained by

$$
P_{\nu}(\xi)=\frac{-1}{2 \pi i} \oint_{\Gamma_{\nu}}\left[A^{0}(\xi)-\lambda I\right]^{-1} d \lambda
$$

where $\Gamma_{\nu}$ is a small circle with centre $\tau_{\nu}|\xi|$. The dimension of the range of $P_{\nu}(\xi)$ is $m_{\nu}$. It follows from (3.5) and (3.6) that $P_{\nu}(\xi)$ is homogeneous of degree zero and

$$
P_{\nu}(-\xi)=P_{k-\nu+1}(\xi), \quad P_{(k+1) / 2}(\xi)=P_{(k+1) / 2}(-\xi) .
$$

We should remark further that $P_{\nu}(\xi)$ are analytic functions of $\xi$ in $\boldsymbol{R}^{n}-\{0\}$.

Now the solution of equation (3.1) with $\lambda$ non-real and $f(x)$ in $\mathscr{H}_{0}$ can be obtained in the following form:

$$
\begin{aligned}
v^{0}(x, \lambda) & =(2 \pi)^{-n / 2} \int_{\boldsymbol{R}^{n}} e^{i x \cdot \xi}\left[A^{0}(\xi)-\lambda I\right]^{-1} \hat{f}(\xi) d \xi \\
& =(2 \pi)^{-n / 2} \sum_{\nu=1}^{k} \int_{\boldsymbol{R}^{n}} \frac{e^{i x \cdot \xi} P_{\nu}(\xi)}{\tau_{\nu}|\xi|-\lambda} \hat{f}(\xi) d \xi .
\end{aligned}
$$

Further the set of plane waves $\left\{e^{i x \cdot \xi} P_{\nu}(\xi)\right\}_{\nu=1,2, \cdots, k}$ provides an eigenfunction expansion associated with $L_{0}$. Namely, if we denote by $E_{0}(\sigma),-\infty<\sigma<\infty$, the resolution of the identity for $L_{0}$, then we have easily from (3.8) and the resolvent equation that

$$
\begin{aligned}
& {\left[\left\{E_{0}(b)-E_{0}(a)\right\} f\right](x)=} \\
& = \begin{cases}(2 \pi)^{-n / 2} \sum_{\nu=1}^{[k / 21} \int_{a<\tau_{\nu}|\xi|<b} e^{i x \cdot \xi} P_{\nu}(\xi) \hat{f}(\xi) d \xi, & (a, b) \subset(0, \infty), \\
(2 \pi)^{-n / 2} \sum_{\nu=1}^{[k / 21} \int_{a<-\tau_{\nu}|\xi|<b} e^{i x \cdot \xi \xi} P_{\nu}(-\xi) \hat{f}(\xi) d \xi, & (a, b) \subset(-\infty, 0) ;\end{cases}
\end{aligned}
$$

(3. 10) $\left[\left\{E_{0}(+0)-E_{0}(-0)\right\} f\right](x)=(2 \pi)^{-n / 2} \int_{\boldsymbol{R}^{R^{2}}} e^{i x \cdot \xi} P_{(k+1) / 2}(\xi) \hat{f}(\xi) d \xi$. 
Next we prove a uniqueness theorem which is already established in [12] (see Chapter VI, Theorem 1.7) when the space dimension $n$ is odd.

Theorem 3.1.5) Suppose that $\sigma$ is in $\boldsymbol{R}^{1}-\{0\}$. Let $v$ be a function in $\mathscr{H}_{0}$ which satisfies

$$
\left(L_{0}-\sigma\right) v=0 \text { for }|x|>\rho .
$$

Then $v$ itself must be zero for $|x|>\rho$.

In order to show this we use results of Ludwig [14] on the Radon transform. Let $\mathscr{R}$ be an operator from $\mathscr{D}_{L^{2}}^{\infty}\left(\boldsymbol{R}^{n}\right)$ into $\mathscr{D}_{L^{2}}^{\infty}\left(\boldsymbol{R}^{1}\right.$; $\left.L^{2}\left(S_{n-1}\right)\right)^{6}$ defined by

$$
[\mathscr{R} f](s, \omega)=(2 \pi)^{(n-2) / 2} \int_{-\infty}^{\infty} e^{i s r} \hat{f}(r \omega) d r \quad\left(s \in \boldsymbol{R}^{1}, \omega \in S_{n-1}\right) .
$$

Then it is known in [14] (Theorem 4.9) that $\mathscr{R}$ is one-to-one bicontinuous map of $\mathscr{D}_{L^{2}}^{\infty}\left(\boldsymbol{R}^{n}\right)$ into $\mathscr{D}_{L^{2}}^{\infty}\left(\boldsymbol{R}^{1} ; L^{2}\left(S_{n-1}\right)\right)$ and of $\mathscr{D}\left(\boldsymbol{R}^{n}\right)$ onto $\mathscr{D}\left(\boldsymbol{R}^{1} ; L^{2}\left(S_{n-1}\right)\right) \cap N^{\perp}$, where $N^{\perp}$ consists of functions $\tilde{f}$ in $\mathscr{D}_{L^{2}}^{\infty}\left(\boldsymbol{R}^{1} ;\right.$ $\left.L^{2}\left(S_{n-1}\right)\right)$ such that

$$
\int_{-\infty}^{\infty} \int_{S_{n-1}} s^{k} Y_{\ell}(\omega) \tilde{f}(s, \omega) d s d \omega=0
$$

for all spherical harmonics $Y_{\ell}(\omega)$ of degree $\ell$ and $k<\ell$, further $f=0$ for $|x|>\rho$ if and only if $\mathscr{R} f=0$ for $|s|>\rho$.

Proof of Theorem 3.1. Since $L_{0}$ is a differential operator of constant coefficient, we can assume that $v$ belongs to $\mathscr{D}_{L^{2}}^{\infty}\left(\boldsymbol{R}^{n}\right)$. It is easy to see that

$$
\mathscr{R}\left(L_{0} f\right)=A^{0}(\omega) \frac{1}{i} \frac{\partial}{\partial s} \mathscr{R} f
$$

5) The same result can be proved without assuming that $A^{0}(\xi)$ is isotropic. It is sufficient to assume that $A_{j}$ are Hermitian, cf., Iwasaki [8], in which is extended the method of Lax and Phillips [12] to derive the local decay of solutions for symmetric hyperbolic systems with dissipative and coercive boundary conditions.

6) $\mathscr{D}_{L^{2}}^{\infty}\left(\boldsymbol{R}^{n}\right)$ is the space of all $C^{\infty}$ functions in $\boldsymbol{R}^{n}$ such that $\mathscr{D}^{\alpha} f \in L^{2}\left(\boldsymbol{R}^{n}\right)$ for every multi-index $\alpha . \mathscr{D}\left(\boldsymbol{R}^{n}\right)$ is the space of all $\boldsymbol{C}^{\infty}$ functions in $\boldsymbol{R}^{n}$ with compact support. $\mathscr{D}_{L^{2}}^{\infty}\left(\boldsymbol{R}^{1} ; L^{2}\left(S_{n-1}\right)\right)$ and $\mathscr{D}\left(\boldsymbol{R}^{1} ; L^{2}\left(S_{n-1}\right)\right)$ are the spaces of $L^{2}\left(S_{n-1}\right)$-valued functions in $\boldsymbol{R}^{1}$ satisfying the above properties, respectively, where $S_{n-1}$ is the unit sphere in $\boldsymbol{R}^{n}$. 
Thus $\tilde{v}=R v$ satisfies the equation

$$
\left\{A^{0}(\omega) \frac{1}{i} \frac{\partial}{\partial s}-\sigma\right\} \tilde{v}(s, \omega)=0 \quad \text { for } \quad|s|>\rho .
$$

Putting $\tilde{v}_{\nu}(s, \omega)=P_{\nu}(\omega) \tilde{v}(s, \omega)$, we obtain

$$
\begin{array}{ll}
\tilde{v}_{\nu}(s, \omega)=\left\{\begin{array}{ll}
n_{1}(\omega) e^{i \sigma s / \tau_{\nu}}, & s>\rho, \\
n_{2}(\omega) e^{i \sigma s / \tau_{\nu}}, & s<-\rho,
\end{array} \quad(\nu \neq(k+1) / 2)\right. \\
\tilde{v}_{(k+1) / 2}(s, \omega)=0, & |s|>\rho .
\end{array}
$$

Since $\tilde{v}$, and hence $\tilde{v}_{v}$, are in $\mathscr{D}_{L^{2}}^{\infty}\left(\boldsymbol{R}^{1} ; L^{2}\left(S_{n-1}\right)\right)$, we conclude that

$$
\tilde{v}(s, \omega)=\sum_{\nu=1}^{k} \tilde{v}_{\nu}(s, \omega)=0 \quad \text { for } \quad|s|>\rho .
$$

To complete the proof of the theorem it remains only to show that $\tilde{v} \in N^{\perp}$, which can be verified if we follow the same line of proof as given by Lax and Phillips [12; VI. Theorem 2.7]. Namely, we infer (3.13) with $\tilde{f}$ replaced by $\tilde{v}$ inductively from the relation

$$
\begin{aligned}
& k(k-1) \cdots(k-j) \iint s^{k-j-1} Y_{\ell}(\omega) A^{0}(\omega)^{j+1} \tilde{v}(s, \omega) d s d \omega \\
& \quad=\sigma k(k-1) \cdots(k-j+1) \iint s^{k-j} Y_{\ell}(\omega) A^{0}(\omega)^{j} \tilde{v}(s, \omega) d s d \omega,
\end{aligned}
$$

that is easily obtained from (3.14).

q.e.d.

3. 2. Elementary solution of the operator $L_{0}-\lambda$.

Let us recall that the matrix $A^{0}(\xi)$ has the following spectral representations :

$$
\left\{A^{0}(\xi)-\lambda I\right\}^{-1}=\sum_{\nu=1}^{k} \frac{P_{\nu}(\xi)}{\tau_{\nu}|\xi|-\lambda} .
$$

From this we see that for each $\lambda$ non-real, the function $\left\{A^{0}(\xi)-\lambda I\right\}^{-1} \in \mathcal{S}_{\xi}{ }^{\prime 7}$ and behaves like $0(1)$ as $|\xi| \rightarrow \infty$. Hence its inverse Fourier transform

(3. 16) $E(x, \lambda)=\mathscr{F}^{-1}\left[\left\{A^{0}(\xi)-\lambda I\right\}^{-1}\right]=(2 \pi)^{-n / 2} \int_{\boldsymbol{R}^{n}} e^{i x \cdot \xi}\left\{A^{0}(\xi)-\lambda I\right\}^{-1} d \xi$ can be defined in $\mathcal{S}_{x}{ }^{\prime}$ and becomes an elementary solution of the operator $L_{0}-\lambda$ :

7) $\mathcal{S}^{\prime}$ is the space of temperate distributions. 


$$
\left(L_{0}-\lambda\right) E(x, \lambda)=\delta(x) I \quad(\delta(x) \text { is the delta function). }
$$

Further, we see that $E(x, \lambda) * f(x)$ defines a bounded operator of $\mathscr{H}_{0}$ into $\mathscr{D}\left(L_{0}\right)$ since

$$
A^{0}(\xi)\left\{A^{0}(\xi)-\lambda I\right\}^{-1} \hat{f}(\xi)=\hat{f}(\xi)+\lambda\left\{A^{0}(\xi)-\lambda I\right\}^{-1} \hat{f}(\xi) \in \hat{\mathcal{H}}_{0} .
$$

These imply that $E(x-y, \lambda)(\lambda$ : non-real) is the resolvent kernel of $L_{0}$ :

$$
\left[\left(L_{0}-\lambda\right)^{-1} f\right](x)=\int_{\boldsymbol{R}^{n}} E(x-y ; \lambda) f(y) d y, \quad f \in \mathcal{H}_{0} .
$$

The following results are due to Matsumura [15].

Theorem 3.2. Let $L_{0}$ satisfy assumption (iv). Then the elementary solution $E(x, \lambda)$ of $L_{0}-\lambda$ obtained for each non-real $\lambda$ by (3.16) has the following properties:

(1) $E(x, \lambda)$ is an analytic function of $(x, \lambda)$ in $\left(\boldsymbol{R}^{n}-\{0\}\right) \times$ $\left(\boldsymbol{C}^{1}-\boldsymbol{R}^{1}\right)$.

(2) For each $x \neq 0$ and arbitrary fixed real $\sigma \neq 0$, the limits

$$
E(x, \sigma \pm i 0)=\lim _{\varepsilon \rightarrow \pm 0} E(x, \sigma+i \varepsilon)
$$

exist, where the convergence is uniform in every compact set of $\boldsymbol{R}^{n}-\{0\}$. Further $E(x, \sigma \pm i 0)$ are continuous functians of $(x, \sigma)$ in $\left(\boldsymbol{R}^{n}-\{0\}\right) \times\left(\boldsymbol{R}^{1}-\{0\}\right)$.

(3) Let $\sigma_{0}$ be any real number $\neq 0$. If we choose $\delta>0$ and $\varepsilon_{0}>0$ sufficiently small, then $E(x, \sigma \pm i \varepsilon)$ behave uniformly in $\sigma \in\left(\sigma_{0}-\delta\right.$, $\left.\sigma_{0}+\delta\right)$ and $\varepsilon \in\left[0, \varepsilon_{0}\right)$ like

$$
E(x, \sigma \pm i \varepsilon)=0\left(|x|^{-(n-1) / 2}\right) \quad \text { as }|x| \rightarrow \infty .
$$

Proof. Assertion (1) is obvious. Assertions (2) and (3) are proved by Matsumura [15; Theorems 2.1 and 2.2] for a restristed case (when $L_{0}$ is elliptic and $m_{\nu}=1, \nu=1,2, \cdots, k$ ). However, the same method is applicable also in our case. Let us sketch the outline of the proof. We will only discuss the case of $\sigma>0$. $E(x, \sigma+i \varepsilon)$ defined by (3.16) can be represented as 
(3.21) $\quad \mathscr{F}^{-1}\left[\left\{A^{0}(\xi)-\lambda I\right\}^{-1}\right]=\mathscr{F}^{-1}\left[\phi_{0}(|\xi|)\left\{A^{0}(\xi)-\lambda I\right\}^{-1}\right]+$

$$
\begin{aligned}
& +\mathscr{F}^{-1}\left[\frac{-1}{\lambda}\left\{1-\phi_{0}(|\xi|)\right\} P_{(k+1) / 2}(\xi)\right]+ \\
& +\sum_{\nu=1}^{[k / 2]} \mathscr{F}^{-1}\left[\frac{\left\{1-\phi_{0}(|\xi|)\right\}\left\{1-\phi_{\nu}(|\xi|)\right\}}{\tau_{\nu}|\xi|-\lambda} P_{\nu}(\xi)+\right. \\
& \left.+\frac{1-\phi_{0}(|\xi|)}{\tau_{k-\nu+1}|\xi|-\lambda} P_{k-\nu+1}(\xi)\right]+ \\
& +\sum_{\nu=1}^{[k / 21} \mathscr{F}^{-1}\left[\frac{\phi_{\nu}(|\xi|)}{\tau_{\nu}|\xi|-\lambda} P_{\nu}(\xi)\right] \equiv J_{1}+J_{2}+J_{3}+J_{4} .
\end{aligned}
$$

Here

$$
\begin{aligned}
& \phi_{0}(r)= \begin{cases}1 & |r| \leq 2 \delta \\
0 & |r| \geq 3 \delta, \quad \text { smooth, }\end{cases} \\
& \phi_{\nu}(r)=\left\{\begin{array}{ll}
1 & \left|r-\sigma_{0} / \tau_{\nu}\right| \leq 2 \delta / \tau_{\nu} \\
0 & \left|r-\sigma_{0} / \tau_{\nu}\right| \geq 3 \delta / \tau_{\nu},
\end{array} \quad\right. \text { smooth. }
\end{aligned}
$$

We have chosen $\delta>0$ so small that the supports of all functions $\phi_{\nu}(r)(\nu=0,1, \cdots,[k / 2])$ are disjoint. $J_{1}, J_{2}\left(J_{2}=0\right.$ if $k$ is even $)$ and $J_{3}$ are continuous functions of $x \in \boldsymbol{R}^{n}-\{0\}, \sigma \in\left(\sigma_{0}-\delta, \sigma_{0}+\delta\right)$ and $\varepsilon \in\left[0, \varepsilon_{0}\right)$, and are bounded for large $|x|$ by any power of $1 /|x|$ (see for example Mizohata [17]). On the other hand, $J_{4}$ is represented as

$$
\begin{gathered}
J_{4}=\sum_{\nu=1}^{[k / 2]}(2 \pi)^{-n / 2} \int_{-\infty}^{\infty} \frac{r^{n-1} \phi_{\nu}(r)}{\tau_{\nu} r-\lambda} K_{\nu}(r x) d r ; \\
K_{\nu}(r x)=\int_{S_{n-1}} e^{i r x \cdot \xi} P_{\nu}(\xi) d S_{\xi} .
\end{gathered}
$$

Without loss of generality, we can assume $x \cdot \xi=x_{n} \cdot \xi_{n}$. We can choose the local coordinate $\left(\eta_{1}, \eta_{2}, \cdots, \eta_{n-1}\right)$ at $(0, \cdots, 0, \pm 1)$ such that $\xi_{n}= \pm\left\{1-\sum \eta_{j}^{2}\right\} \quad$ (the Morse transformation). Then, using the argument given in the appendix of [15], we get

$$
\begin{aligned}
& K_{\nu}(r x)=\operatorname{const}\left(\frac{2 \pi}{r|x|}\right)^{(n-1) / 2} e^{i r|x|} P_{\nu}\left(\frac{x}{|x|}\right)+ \\
& \quad+\operatorname{const}\left(\frac{2 \pi}{r|x|}\right)^{(n-1) / 2} e^{-i r|x|} P_{\nu}\left(-\frac{x}{|x|}\right)+q_{\nu}(r x),
\end{aligned}
$$

where $q_{\nu}(x)$ is estimated for large $|x|$ as

$$
\left|q_{\nu}(x)\right|+\sum_{j=1}^{n}\left|\frac{\partial q_{\nu}}{\partial x_{j}}(x)\right| \leq \text { const }|x|^{-(n+1) / 2} .
$$


Substituting (3.23) in (3.22), and putting $\psi_{\nu}(r)=r^{(n-1) / 2} \phi_{\nu}(r)$ and $q_{\nu, x}(r)=r^{n-1} \phi_{\nu}(r) q_{\nu}(r x)$, we have

$$
\text { (3. 25) } \begin{aligned}
J_{4}= & \sum_{\nu=1}^{[k / 21} \text { const }|x|^{-(n-1) / 2} P_{\nu}(\theta) \int_{-\infty}^{\infty} \frac{e^{i r|x|} \psi_{\nu}(r)}{\tau_{\nu} r-(\sigma \pm i \varepsilon)} d r+ \\
& +\sum_{\nu=1}^{[k / 2]} \operatorname{const}|x|^{-(n-1) / 2} P_{\nu}(-\theta) \int_{-\infty}^{\infty} \frac{e^{-i r|x|} \psi_{\nu}(r)}{\tau_{\nu} r-(\sigma \pm i \varepsilon)} d r+ \\
& +\sum_{\nu=1}^{[k / 21} \int_{-\infty}^{\infty} \frac{q_{\nu, x}(r)}{\tau_{\nu} r-(\sigma \pm i \varepsilon)} d r \equiv I_{1}+I_{2}+I_{3}, \quad \theta=x /|x| .
\end{aligned}
$$

Note the relations

$$
\int_{-\infty}^{\infty} \frac{e^{i r|x|}}{r-(\sigma \pm i \varepsilon)} d r= \pm 2 \pi i Y( \pm|x|) e^{i(\sigma \pm i \varepsilon|x|} \quad(\varepsilon>0)
$$

where $Y(\rho)$ is the Heaviside function: $Y(\rho)=1$ if $\rho \geq 0,=0$ if $\rho<0$. Then we get

$$
\begin{aligned}
I_{1}+I_{2}= & \sum_{\nu=1}^{[k / 21} c_{\nu}^{ \pm}(x, \sigma, \varepsilon)|x|^{-(n-1) / 2} P_{\nu}( \pm \theta)+ \\
& +\sum_{\nu=1}^{[k / 21} d_{\nu}^{ \pm}(x, \sigma, \varepsilon)|x|^{-(n-1) / 2} P_{\nu}(\mp \theta),
\end{aligned}
$$

where

$$
\left\{\begin{array}{l}
c_{\nu}^{ \pm}(x, \sigma, \varepsilon)=\text { const } \tau_{\nu}^{-1} \int_{-\infty}^{|x|} e^{ \pm i(\sigma \pm i \varepsilon)(|x|-\rho) / \tau_{\nu}} \hat{\psi}_{\nu}(\rho) d \rho, \\
d_{\nu}^{ \pm}(x, \sigma, \varepsilon)=\text { const } \tau_{\nu}^{-1} \int_{|x|}^{\infty} e^{\mp i(\sigma \pm i \varepsilon)(|x|-\rho) / \tau} \nu \hat{\psi}_{\nu}(\rho) d \rho .
\end{array}\right.
$$

Since $\hat{\psi}_{\nu}(\rho)=\left[\mathscr{F}_{\psi} \psi_{\nu}\right](\rho)$ is rapidly decreasing in $|\rho|$, we see that $c_{\nu}^{ \pm}(x, \sigma, \varepsilon)=0(1)$ and $d_{\nu}^{ \pm}(x, \sigma, \varepsilon)=0\left(|x|^{-1}\right)$ uniformly in $\sigma$ and $\varepsilon$. On the other hand, since we have

$$
I_{3}=\sum_{\nu=1}^{[k / 2]}\left\{\int_{-\infty}^{\infty} \frac{q_{\nu, x}(r)-q_{\nu, x}(\sigma)}{\tau_{\nu} r-(\sigma \pm i \varepsilon)} d r+\frac{q_{\nu, x}(\sigma)}{\pi i \tau_{\nu}}\right\}
$$

it follows easily from (3.24) that $I_{3}=0\left(|x|^{-(n-1) / 2}\right)$. Summing up we conclude assertions (2) and (3).

q. e. d.

Using relations $(3.21) \sim(3.29)$ given by Matsumura, we can prove additionally the following estimates of $E(x, \sigma \pm i \varepsilon)$.

Theorem 3.3. (1) Let $\sigma_{0}, \delta$ and $\varepsilon_{0}$ be as given in the above theorem, and define the orthogonal projections $P_{+}(\xi)$ and $P_{-}(\xi)$ in $\boldsymbol{C}^{m}$ as follows : 


$$
P_{ \pm}(\xi)=\sum_{\nu=1}^{[k / 2]} P_{\nu}( \pm \xi)
$$

Then $\left\{I-P_{ \pm}(\theta)\right\} E(x, \sigma \pm i \varepsilon)$ behave uniformly in $\sigma \in\left(\sigma_{0}-\delta, \sigma_{0}+\delta\right)$ and $\varepsilon \in\left[0, \varepsilon_{0}\right)$ like

$$
\left\{I-P_{ \pm}(\theta)\right\} E(x, \sigma \pm i \varepsilon)=0\left(|x|^{-(n+\gamma) / 2}\right) \quad \text { as }|x| \rightarrow \infty,
$$

where $\gamma$ is any constant such that $0<\gamma<1$, and $\theta=x /|x|$.

(2) $E(x, \sigma \pm i \varepsilon)$ are Hölder continuous in $\sigma \neq 0$, and we have the following estimates uniformly in $\sigma, \sigma^{\prime} \in\left(\sigma_{0}-\delta, \sigma_{0}+\delta\right)$ and $\varepsilon \in\left[0, \varepsilon_{0}\right)$.

(3. 32) $\left|E(x, \sigma \pm i \varepsilon)-E\left(x, \sigma^{\prime} \pm i \varepsilon\right)\right| \leq$ const $|x|^{-(n-3) / 2}\left|\sigma-\sigma^{\prime}\right|^{h}$,

where $h$ is any constant such that $0<h<1$, and the "const" is independent of $x$ (large) and $\varepsilon$.

Proof. In expression (3.21), we see easily that $J_{1}, J_{2}$ and $J_{3}$ are differentiable in $\sigma$ and their derivatives are also majorized by any power of $1 /|x|$ at infinity. Hence we have only to show estimates (3.31) and (3.32) for $J_{4}=I_{1}+I_{2}+I_{3}$ (cf. (3.25)). Since the coefficients $c_{\nu}^{ \pm}(x, \sigma, \varepsilon)$ and $d_{\nu}^{ \pm}(x, \sigma, \varepsilon)$ are scalar functions, we have from (3.27) and (3.28)

$$
\left\{I-P_{ \pm}(\theta)\right\}\left(I_{1}+I_{2}\right)=0\left(|x|^{-(n+1) / 2}\right),
$$

On the other hand, we have easily from (3.24)

$$
\begin{aligned}
& q_{\nu, x}(\sigma)=0\left(|x|^{-(n+1) / 2}\right) ; \\
& \left|q_{\nu, x}(\sigma)-q_{\nu, x}\left(\sigma^{\prime}\right)\right| \leq \mathrm{const}|x|^{-(n+\infty) / 2}\left|\sigma-\sigma^{\prime}\right|^{(1-\alpha) / 2} \quad(-1<\alpha<1) .
\end{aligned}
$$

Since the support of $q_{v, x}(\sigma)$ is compact uniformly in $x$ (large), we have, using Hilfsatz 1.3 of Friedrichs [3], that

$$
I_{3} \equiv I_{3}(x, \sigma, \varepsilon)=\sum_{\nu=1}^{[k / 2]} \int_{-\infty}^{\infty} \frac{q_{\nu, x}(r)}{\tau_{\nu} r-(\sigma \pm i \varepsilon)} d r
$$

has the following properties:

$$
I_{3}(x, \sigma, \varepsilon)=0\left(|x|^{-(n+\alpha) / 2}\right) ;
$$

$$
\left|I_{3}(x, \sigma, \varepsilon)-I_{3}\left(x, \sigma^{\prime}, \varepsilon\right)\right| \leq \text { const }|x|^{-(n+\alpha) / 2}\left(\sigma-\sigma^{\prime}\right)^{\left(1-\alpha^{\prime}\right) / 2},
$$

where $\alpha^{\prime}$ may be any constant such that $\alpha^{\prime}<\alpha$. (3.33) and (3.34) imply (3.31). (3.32) follows easily from (3.35) if we note that $I_{1}$ 
and $I_{2}$ are differentiable in $\sigma$ and their derivatives are estimated by const $|x|^{-(n-3) / 2}$. The last estimate is evident since $|x|^{-1}(|x|-\rho) \hat{\psi}_{2}(\rho)$ is rapidly decreasing in $|\rho|$ uniformly in $|x|>1$.

Now we return to equation (3.1). As is shown by (3.18), this equation with $\lambda=\sigma+i \varepsilon(\varepsilon \neq 0)$ has a unique solution

$$
v^{0}(x, \sigma+i \varepsilon)=\int_{\boldsymbol{R}^{n}} E(x-y, \sigma+i \varepsilon) f(y) d y \in \mathscr{H}_{0}
$$

for each $f \in \mathscr{H}_{0}$. By (1) and (2) of Theorem 3.2, the limits

$$
v^{0}(x, \sigma \pm i 0)=\lim _{\varepsilon \rightarrow \pm 0} \int_{\boldsymbol{R}^{n}} E(x-y, \sigma+i \varepsilon) f(y) d y
$$

exist for each $\sigma \neq 0$, and are distribution solutions of (3.1) with $\lambda=\sigma$.

We can easily verify the following corollary of Theorems 3.2 and 3.3.

Corollary 3.1. Let $\rho$ be any positive number. Then for each function $f(x)$ in $\mathscr{H}_{0}$ with support in the ball $K_{\mathrm{p}}=\{|x|<\rho\}$, the functions

$$
v^{0}(x, \sigma \pm i \varepsilon)=\int_{\boldsymbol{R}^{n}} E(x-y, \sigma \pm i \varepsilon) f(y) d y \quad(\varepsilon \geq 0,|x|>\rho)
$$

satisfy the following asymptotic estimates as $|x| \rightarrow \infty$ :

$$
\begin{gathered}
\left|v^{0}(x, \sigma \pm i \varepsilon)\right| \leq C\left(K_{\rho}\right)|x|^{-(n-1) / 2}|| f \|_{0, K_{\rho}} \\
\left|\left\{I-P_{ \pm}\left(\frac{x}{|x|}\right)\right\} v^{0}(x, \sigma \pm i \varepsilon)\right| \leq C\left(K_{\rho}\right)|x|^{-(n+\gamma) / 2}|| f \|_{0, K_{\rho}},
\end{gathered}
$$

where $\gamma$ is any constant such that $0<\gamma<1$, and $C\left(K_{\rho}\right)$ is a positive constant which is independent of $f$ and $\varepsilon$. Further, $v^{0}(x, \sigma \pm i \varepsilon)$ are Hölder continuous in $\sigma \neq 0$; that is, for each $\sigma \neq 0$ there exists $\delta>0$ independent of $\varepsilon$ such that

$$
\left|v^{0}(x, \sigma \pm i \varepsilon)-v^{0}\left(x, \sigma^{\prime} \pm i \varepsilon\right)\right| \leq C\left(K_{\rho}\right)|x|^{-(n-3) / 2}\left|\sigma-\sigma^{\prime}\right|{ }^{h}|| f \|_{0, K_{\rho}}
$$

if $\left|\sigma-\sigma^{\prime}\right|<\delta$, where $h$ is any constant such that $0<h<1$.

Proof. Inequalities (3.36) and (3.38) follow easily from (3.20) and (3.32), respectively. In order to verify (3.37), we put 


$$
\begin{aligned}
& \left\{I-P_{ \pm}\left(\frac{x}{|x|}\right)\right\} v^{0}(x, \sigma \pm i \varepsilon)= \\
& =\int_{\boldsymbol{R}^{n}}\left\{I-P_{ \pm}\left(\frac{x-y}{|x-y|}\right)\right\} E(x-y, \sigma \pm i \varepsilon) f(y) d y+ \\
& \quad+\int_{\boldsymbol{R}^{n}}\left\{P_{ \pm}\left(\frac{x-y}{|x-y|}\right)-P_{ \pm}\left(\frac{x}{|x|}\right)\right\} E(x-y, \sigma \pm i \varepsilon) f(y) d y .
\end{aligned}
$$

Then, from (3.31), the first term of the second member can be estimated as $0\left(|x|^{-(n+\gamma) / 2}\right)\|f\|_{0, K_{\rho}}$. On the other hand, since $P_{ \pm}(\xi)$ are smooth in $\xi(\neq 0)$, we have

$$
\begin{gathered}
\left|P_{ \pm}\left(\frac{x-y}{|x-y|}\right)-P_{ \pm}\left(\frac{x}{|x|}\right)\right| \leq \max _{|\xi|=1} \sum_{j=1}^{n}\left|\frac{\partial P_{ \pm}}{\partial \xi_{j}}(\xi)\right| \cdot\left|\frac{x-y}{|x-y|}-\frac{x}{|x|}\right| \\
\leq \text { const }|y| /|x| .
\end{gathered}
$$

This shows with (3.20) that the second term is estimated by $0\left(|x|^{-(n+1) / 2}\right)\|f\|_{0, K_{\rho}}$. Thus we have (3.37).

\section{The Spectrum of the Perturbed Operator $L_{1}$}

4.1. Discreteness of the point spectrum and the radiation conditions.

Suppose that $\sigma(\neq 0)$ is real, and let us consider the following eigenvalue problem in $\mathscr{H}_{1}$ :

$$
L_{1} \varphi-\sigma \varphi=0, \quad \varphi \in \mathcal{H}_{1} .
$$

Since $\sigma \neq 0$, every non-trivial solution of (4.1) lies in the orthogonal complement of the null space of $L_{1}$; i. e.,

$$
\varphi=\varphi(\cdot, \sigma) \in \mathscr{D}\left(L_{1}\right) \ominus \mathscr{M}\left(L_{1}\right) \text {. }
$$

We have assumed that outside of the ball $K_{\rho}=\{|x|<\rho\}, M(x)=I$ and $L=L_{0}$. Thus we can write (4.1) in the form :

$$
L_{0} \varphi(x, \sigma)-\sigma \varphi(x, \sigma)=0 \text { for }|x|>\rho .
$$

Theorem 4.1. The point spectrum of $L_{1}$ consists of isolated eigenvalues which are of finite multiplicity except the origin 0 . Further, all eigenfunctions corresponding to non-zero eigenvalues are of bounded supports contained in the ball $K_{\rho}=\{|x|<\rho\}$.

Proof. The second assertion is a direct consequence of 
Theorem 3.1. To show the discreteness of the point spectrum we use Lemma 2.1. Let $\left\{\sigma_{n}\right\}$ be a sequence of non-zero eigenvalues which converges as $n \rightarrow \infty$ to a finite $\sigma$, and let $\left\{\varphi_{n}\right\}$ be a set of corresponding eigenfunctions such that

$$
\left(\varphi_{m}, \varphi_{n}\right)_{1}=\int_{G} M(x) \varphi_{m}(x) \cdot \overline{\varphi_{n}(x)} d x=\delta_{m n}
$$

Then, since $\left\|\rho_{n}\right\|_{1}+\left\|L_{1} \varphi_{n}\right\|_{1} \leq 1+\sup _{n}\left|\sigma_{n}\right|$, combining inequality (2.10) with the Rellich compactness criterion we deduce that there exists a subsequence $\left\{\varphi_{n^{\prime}}\right\}$ of $\left\{\varphi_{n}\right\}$ which converges to a function $\varphi$ as $n^{\prime} \rightarrow \infty$ in the sense of $L^{2}$-norm over any bounded subdomain of $\bar{G}$. On the other hand, we know that the supports of $\varphi_{n}$ are all contained in the ball $K_{\rho}$. Thus $\varphi_{n}{ }^{\prime}$ must converges to $\varphi$ in $\mathscr{H}_{1}$ as $n^{\prime} \rightarrow \infty$, which contradicts (4.4).

q. e. d.

Remark 4. 1. If the operator $L-\sigma M(x)(\sigma \neq 0)$ has the unique continuation property, then from the second assertion of the above theorem we see that the operator $L_{1}$ has no eigenvalue except the origin 0 . However, the origin 0 is somewhat ambiguous; the possibility of the origin 0 being an eigenvalue remains even if $L_{1}$ is assumed to be elliptic.

Now we give a formulation of the radiation conditions attached to the operator $L_{1}$ (cf., [18]).

Definition 4.1. ${ }^{8}$ Let $f(x)$ be a $\boldsymbol{C}^{m}$-valued function defined for large $|x|$. Then $f$ is said to satisfy the incoming radiation condition if it satisfies the following properties:

$$
\left\{\begin{array}{l}
f(x)=0\left(|x|^{-(n-1) / 2}\right) ; \\
\left\{I-P_{+}\left(\frac{x}{|x|}\right)\right\} f(x)=0\left(|x|^{-(n+\gamma) / 2}\right) \quad(\gamma>0),
\end{array}\right.
$$

and $f$ is said to satisfy the outgoing radiation condition if it satisfies the following properties:

8) The radiation condition plays a role of a boundary condition at infinity in the problem (4.9) or (4.13) given below. So we may have several different formulations of the radiation condition. In fact, our formulation differs from that given by Lax and Phillips [12], where they restrict the behaviors of functions at infinity by introducing the initially incoming and eventually outgoing subspaces. 


$$
\left\{\begin{array}{l}
f(x)=0\left(|x|^{-(n-1) / 2}\right) ; \\
\left\{I-P_{-}\left(\frac{x}{|x|}\right)\right\} f(x)=0\left(|x|^{-(n+\gamma) / 2}\right) \quad(\gamma>0),
\end{array}\right.
$$

where $P_{ \pm}(\xi)$ are the projections in $C^{m}$ defined by (3.30).

Remark 4.2. The functions $v^{0}(x, \sigma+i \varepsilon)$ and $v^{0}(x, \sigma-i \varepsilon)(\varepsilon \geq 0)$ in Corollary 3.1 satisfy the incoming and outgoing radiation conditions, respectively.

It is obvious that every $L^{2}$-eigenfunction of $L_{1}$ corresponding to a non-zero eigenvalue satisfies both the incoming and outgoing radiation conditions since it is a function of bounded support.

We denote by $\mathscr{D}_{\text {loc }}\left(L_{1}\right)$ the following class of functions :

$$
\begin{array}{r}
\mathscr{D}_{l o c}\left(L_{1}\right)=\left\{f \in\left[L_{l o c}^{2}(G)\right]^{m} ;\left\{1-\beta_{r}(|x|)\right\} f \in \mathscr{D}\left(L_{1}\right)\right. \\
\text { for any } r>\rho\},
\end{array}
$$

where $\beta_{r}(s)$ is a $C^{\infty}$ scalar function such that

$$
\beta_{r}(s)= \begin{cases}0 & s<r \\ 1 & s>r+1\end{cases}
$$

and we denote by $L_{1, \text { loc }}$ the operator acting in $\mathscr{D}_{\text {loc }}\left(L_{1}\right)$ defined by

$$
L_{1, l o c} f=L_{1} f \quad \text { locally on } \mathscr{D}_{\text {loc }}\left(L_{1}\right) \text {. }
$$

Lemma 4.1. For a non-zero real number $\sigma$, if there exists a non-trivial solution $\phi(x, \sigma)$ in $\mathscr{D}_{\text {loc }}\left(L_{1}\right)$ of the equation

$$
\left(L_{1, l o c}-\sigma\right) \varphi=0,
$$

which satisfies the incoming or outgoing radiation condition (4.5) then the solution $\varphi$ belongs to $\mathcal{H}_{1}$, and hence this $\sigma$ is an eigenvalue of $L_{1}$.

Proof. We have only to deal with case when $\varphi$ satisfies the incoming radiation condition. We put $G_{r}=\{x \in G ;|x|<r\}(r>\rho)$. Then using the Green formula we get

$$
\begin{aligned}
0 & =\int_{G_{r}}\{(L-\sigma M(x)) \varphi(x) \cdot \overline{\varphi(x)}-\varphi(x) \cdot \overline{(L-\sigma M(x)) \varphi(x)}\} d x \\
& =-\frac{1}{i} \int_{|x|=r} A^{0}\left(\frac{x}{|x|}\right) \varphi(x) \cdot \overline{\varphi(x)} d S_{x} .
\end{aligned}
$$


Since

$$
\sum_{\nu=1}^{[k / 2]} P_{\nu}\left(\frac{x}{|x|}\right) \oplus \sum_{\nu=1}^{[k / 2]} P_{\nu}\left(-\frac{x}{|x|}\right)=P_{+}\left(\frac{x}{|x|}\right) \oplus P_{-}\left(\frac{x}{|x|}\right)=I \ominus P_{(k+1) / 2}\left(\frac{x}{|x|}\right),
$$

and

$$
\begin{aligned}
& A^{0}\left(\frac{x}{|x|}\right) P_{\nu}\left( \pm \frac{x}{|x|}\right)= \pm \tau_{\nu} P_{2}\left( \pm \frac{x}{|x|}\right) \quad(\nu=1,2, \cdots,[k / 2]) ; \\
& A^{0}\left(\frac{x}{|x|}\right) P_{(k+1) / 2}\left(\frac{x}{|x|}\right)=0
\end{aligned}
$$

it follows that

$$
\begin{aligned}
0=\sum_{\nu=1}^{[k / 21} \tau_{\nu} \int_{|x|=r} P_{\nu}\left(\frac{x}{|x|}\right) \varphi(x) \cdot \overline{\varphi(x)} d S_{x} \\
\quad-\sum_{\nu=1}^{[k / 21} \tau_{\nu} \int_{|x|=r} P_{\nu}\left(-\frac{x}{|x|}\right) \varphi(x) \cdot \overline{\varphi(x)} d S_{x} \equiv J_{1}-J_{2},
\end{aligned}
$$

where

$$
\begin{gathered}
J_{1} \geq \tau_{[k / 2]} \int_{|x|=r} P_{+}\left(\frac{x}{|x|}\right) \varphi(x) \cdot \overline{\varphi(x)} d S_{x}=\tau_{[k / 2]}\left\|P_{+}\left(\frac{x}{|x|}\right) \varphi(x)\right\|_{L^{2}\left(S_{r}\right)}^{2} ; \\
J_{2} \leq \tau_{1} \int_{|x|=r} P_{-}\left(\frac{x}{|x|}\right) \varphi(x) \cdot \overline{\varphi(x)} d S_{x} \\
\leq \tau_{1}\left\|\left\{I-P_{+}\left(\frac{x}{|x|}\right)\right\} \varphi(x)\right\|_{L^{2}\left(S_{r}\right)}^{2}=0\left(|x|^{-1-\gamma}\right),
\end{gathered}
$$

by $(4.5)_{-\uparrow}$, where $S_{r}=\{|x|=r\}$. Hence we get noting $J_{1}=J_{2}$

$$
\begin{gathered}
\int_{|x|=\gamma}|\varphi(x)|^{2} d S_{x}=\left\|P_{+}\left(\frac{x}{|x|}\right) \varphi(x)\right\|_{L^{2}\left(S_{r}\right)}^{2}+\left\|\left\{I-P_{+}\left(\frac{x}{|x|}\right)\right\} \varphi(x)\right\|_{L^{2}\left(S_{r}\right)}^{2} \\
\leq \text { const } J_{1}+0\left(|x|^{-1-\gamma}\right)=0\left(|x|^{-1-\gamma}\right) .
\end{gathered}
$$

Since $\gamma>0$, this proves that $\varphi(x)$ belongs to $\mathcal{H}_{1}$.

q. e. d.

\section{2. Absolute continuity of the continuous spectrum.}

Let $v_{\varepsilon}=v_{\varepsilon}(x, \sigma)$ be the solution in $\mathscr{H}_{1}$ of the equation

$$
L_{1} v_{\varepsilon}-(\sigma+i \varepsilon) v_{\varepsilon}=f(x) \quad\left(\sigma, \varepsilon \in \boldsymbol{R}^{1}-\{0\}\right),
$$

where $f(x)$ is any function in $\mathscr{H}_{1}$ with compact support in $\bar{G}_{r}(r>\rho)$. Let $\beta_{r}(s)$ be the function given by (4.7), and put $w_{\varepsilon}=\beta_{r}(|x|) v_{\varepsilon}$. Then $w_{\varepsilon}$ is in $\mathcal{H}_{0}$ and satisfies the equation 


$$
L_{0} w_{\varepsilon}-(\sigma+i \varepsilon) w_{\mathrm{\varepsilon}}=\frac{1}{i} \beta_{r}^{\prime}(|x|) A^{0}\left(\frac{x}{|x|}\right) v_{\mathrm{\varepsilon}}(x, \sigma) .
$$

$\beta_{r}{ }^{\prime}$ means the derivative of $\beta_{r}$ and is a function with support in $r<s<r+1$. Thus, as was studied in $\S 3, v_{\varepsilon}(x, \sigma)$ can be expressed for $|x|>r+1$ in the form:

(4.12) $v_{\varepsilon}(x, \sigma)=\frac{1}{i} \int_{R^{n}} E(x-y, \sigma+i \varepsilon) A^{0}\left(\frac{y}{|y|}\right) \beta_{r}^{\prime}(y) v_{\varepsilon}(y, \sigma) d y$

if we note that $v_{\mathrm{g}}=w_{\mathrm{g}}$ for $|x|>r+1$.

In the following of this section, we shall derive that the limit $v_{ \pm}$as $\varepsilon \rightarrow \pm 0$ of $v_{\varepsilon}$ exists in the class of functions which satisfy the radiation conditions $(4.5)_{ \pm}$if $\sigma$ is not an eigenvalue of $L_{1}$, and satisfies the equation

$$
L_{1, l_{o c}} v_{ \pm}-\sigma v_{ \pm}=f(x) \text {. }
$$

Following Éìdus [2; Theorem 4], we introduce the Hilbert space $\mathcal{K}_{1+\delta}(\delta>0)$ of functions $f$ in $\mathscr{H}_{1, \text { loc }}$ such that $(1+|x|)^{-(1+\delta) / 2} f(x) \in$ $\mathscr{H}_{1}$, where the inner product and the norm of $\mathcal{K}_{1+\delta}$ are defined respectively by

$$
[f, g]_{1+\delta}=\int_{G} \frac{M(x) f(x) \cdot \overline{g(x)}}{(1+|x|)^{1+\delta}} d x \quad \text { and } \quad|f|_{1+\delta}=[f, f]_{1+\delta}^{1 / 2} .
$$

Definition 4.2. A set $V$ of functions in $\mathcal{K}_{1+\delta}$ is said to be nondissipating if given any $\alpha>0$ there exists a positive constant $R=R(\alpha)$ such that

$$
\sup _{f \in V} \int_{! x \mid>R} \frac{M(x) f(x) \cdot \overline{f(x)}}{(1+|x|)^{1+\delta}} d x<\alpha .
$$

Lemma 4.2. Let $V$ be a set of functions in $\mathscr{D}\left(L_{1}\right) \ominus \mathscr{N}\left(L_{1}\right)$ such that

$$
\sup _{f \in V}\left\{|f|_{1+\delta}+\left|L_{1} f\right|_{1+\delta}\right\}<\infty .
$$

If $V$ is non-dissipating, then $V$ is pre-compact in $\mathcal{K}_{1+\delta}$ : For any sequence $\left\{f_{n}\right\} \subset V$ there exists a subsequence $\left\{f_{n^{\prime}}\right\}$ and $f \in \mathcal{K}_{1+\delta} \cap$ $\mathscr{D}_{\text {loc }}\left(L_{1}\right)$ such that 


$$
\left|f_{n^{\prime}}-f\right|_{1+\delta} \rightarrow 0
$$

$$
\left(n^{\prime} \rightarrow \infty\right)
$$

Proof. From (4.16) and (2.10), we see that $V$ forms a bounded set in $\left[\mathcal{E}_{L^{2}}^{1}\left(G_{r}\right)\right]^{m}(r>R){ }^{9)}$ Every bounded set in $\left[\mathcal{E}_{L^{2}}^{1}\left(G_{r}\right)\right]^{m}$ is precompact with respect to its weak topology, and to the $L^{2}$-norm over $G_{r}$ by the Rellich compactness criterion. Thus the lemma is proved if we note inequality (4.15).

q. e. d.

Remark 4. 3. In case the operator $L$ is elliptic and the coercivity condition on $N(z)$ is taken as in Remark 2.2, the above assertion holds for any set $V$ in $\mathcal{K}_{1+\delta}$ satisfying (4.15) and (4.16).

Lemma 4. 3. Let $v_{\varepsilon}$ be the solution of (4.10) with a fixed $\sigma$, and put $V=\left\{v_{\varepsilon} ; 0<\varepsilon<1\right\}$ (or $=\left\{v_{\varepsilon} ; 0>\varepsilon>-1\right\}$ ). If $V$ is bounded in $\mathcal{H}_{1, \text { loc }}$, then $V$ forms a non-dissipating bounded set in $\mathcal{K}_{1+\delta}$. Further, if the limit $v_{+}$(resp. $\left.v_{-}\right)$as $\varepsilon \rightarrow+0(-0)$ of $v_{\varepsilon}$ exists in $\mathcal{H}_{1, \text { loc }}$, then $v_{+}\left(v_{-}\right)$satisfies (4.13) and the incoming (outgoing) radiation condition at infinity.

Proof. Since $v_{\varepsilon}$ is expressed by (4.12) for $|x|$ large, it follows from Corollary 3.1 that

$$
\left|v_{\varepsilon}(x)\right| \leq C_{r}|x|^{-(n-1) / 2}|| v_{\varepsilon} \|_{1, G_{r}} .
$$

Thus, if $\left\|v_{\varepsilon}\right\|_{1, G_{r}}$ is bounded, then $v_{\varepsilon} \in \mathcal{K}_{1+\delta}$ and satisfies (4.15) with $R$ independent of $\varepsilon$. Further, if the limit $v_{+}$exists, then by (4.12) it can be expressed for $|x|$ large in the form

$$
v_{+}(x)=\frac{1}{i} \int_{\boldsymbol{R}^{n}} E(x-y ; \sigma+i 0) A^{0}\left(\frac{y}{|y|}\right) \beta_{r}^{\prime}(|y|) v_{+}(y) d y
$$

As has been already mentioned in Remark 4.2, the right member of (4.19) satisfies the radiation condition.

q. e. d.

We can now prove the following theorem.

Theorem 4. 2. Suppose that $\sigma \notin \sigma_{p}\left(L_{1}\right) \cup\{0\}$ and $f(x)$ is any func-

9) $\mathcal{E}_{L^{2}}^{1}\left(G_{r}\right)$ is the space of all functions such that $D^{\alpha} f \in L^{2}\left(G_{r}\right),|\alpha| \leq 1$, with norm: $\left(\sum_{|\alpha| \leq 1}\left\|D^{\alpha} f\right\|_{G_{r}}^{2}\right)^{1 / 2}$. 
tion in $\mathscr{H}_{1}$ which vanishes for $|x|>r(>\rho)$. Then, as $\varepsilon \rightarrow \pm 0$, the solution $v_{\varepsilon}$ of equation (4.10) tends in $\mathcal{K}_{1+\delta}$, i.e., in the sense of the local energy norm, to the solution $v_{ \pm}$of equation (4.13) $)_{ \pm}$satisfying the radiation condition $(4.5)_{ \pm}$.

Proof. (Cf., Éídus [2]; Theorem 4) We carry out the proof for the case of $\varepsilon>0$. We first establish the fact that $\left\{v_{\varepsilon} ; 0<\varepsilon<1\right\}$ forms a bounded set in $\mathcal{K}_{1+\delta}$. If we assume the contrary, then $\left|v_{\varepsilon_{n}}\right|_{1+\delta} \rightarrow \infty$ for some sequence $\varepsilon_{n} \rightarrow+0$. We put $\tilde{v}_{\varepsilon}=\left|v_{\varepsilon}\right|_{1+\delta}^{-1} v_{\varepsilon}$ (below we shall omit the index $n$ ). Then $|\tilde{v}|_{1+\delta}=1$ and

$$
L_{1} \tilde{v}_{\varepsilon}=(\sigma+i \varepsilon) \tilde{v}_{\varepsilon}+\left|v_{\varepsilon}\right|_{1+\delta}^{-1} f .
$$

Thus $\left|\tilde{v}_{\varepsilon}\right|_{1+\delta}+\left|L_{1} \tilde{v}_{\varepsilon}\right|_{1+\delta}$ is bounded in $\varepsilon$, and thus by Lemma 4.3 $\left\{\tilde{v}_{\varepsilon}\right\}$ is non-dissipating. Let $Q_{1}$ denote the projection onto $\Re\left(L_{1}\right)$, and put $\tilde{v}_{\varepsilon}=\tilde{u}_{\varepsilon}+Q_{1} \tilde{v}_{\varepsilon}$. Then by (4.20) $Q_{1} \tilde{v}_{\varepsilon}=\left|v_{\varepsilon}\right|{ }_{1+\delta}^{-1} Q_{1} f /(\sigma+i \varepsilon)$, and hence $\rightarrow 0$ in $\mathcal{K}_{1+\delta}$ as $\varepsilon \rightarrow+0$. These imply that the set $V=\left\{\tilde{u}_{\varepsilon}\right\}$ satisfies the conditions of Lemma 4.2. Thus, it follows from (4.20) and Lemma 4.2 that there exists a solution $u_{+}$of

$$
L_{1, l o c} \tilde{u}_{+}=\sigma \tilde{u}_{+}
$$

such that $\left|\tilde{u}_{+}\right|_{1+\delta}=1$, if we note that $\left|v_{\varepsilon}\right|_{1+\delta}^{-1} \rightarrow 0(\varepsilon \rightarrow+0)$. On the other hand, Lemma 4.3 asserts that this $u_{+}$satisfies the incoming radiation condition. It follows from Lemma 4.1 that $\sigma$ must be an eigenvalue of $L_{1}$, and this contradicts our assumption on $\sigma$. Thus we have proved the inequality

$$
\left|v_{\varepsilon}\right|_{1+\delta}<C<\infty .
$$

Using this inequality, we apply the same argument to $\left\{v_{\varepsilon}\right\}$ as was applied to $\left\{\tilde{v}_{\varepsilon}\right\}$. Then we find that we can select a subsequence $\left\{v_{\varepsilon}\right\}$ from the $v_{\varepsilon}$, where $\varepsilon_{n} \rightarrow+0$, which converges in $\mathscr{K}_{1+\delta}$ to some function $v_{+}$of the form $u_{+}-\sigma^{-1} Q_{1} f$ satisfying (4.13) $)_{+}$and (4.5)+. Since $\sigma$ is not in $\sigma_{p}\left(L_{1}\right) \cup\{0\}$, in view of Lemma 4.1, we see that not only does $v_{\varepsilon n} \rightarrow v_{+}$as $\varepsilon_{n} \rightarrow+0$, but also $v_{\varepsilon} \rightarrow v_{+}$. This proves the theorem.

q. e. d.

By the same argument as above, it is not difficult to show that $v_{\varepsilon}(\cdot, \mu) \rightarrow v_{ \pm}(\cdot, \sigma)$ as $\varepsilon \rightarrow \pm 0$ and $\mu \rightarrow \sigma$. Thus, if we choose the interval $[a, b]$ such that any point of $\sigma_{p}\left(L_{1}\right) \cup\{0\}$ is not in $[a, b]$, 
then, as $\varepsilon \rightarrow \pm 0, \quad v_{s}(\cdot, \sigma)$ tends in $\mathcal{K}_{1+\delta}$ to $v_{ \pm}(\cdot, \sigma)$ uniformly with respect to $\sigma \in[a, b]$. This implies that $v_{ \pm}(\cdot, \sigma)$ are continuous functions of $\sigma \in[a, b]$ with values in $\mathcal{K}_{1+\hat{\delta}}$.

Corollary 4.1. Let $E_{1}(\sigma)(-\infty<\sigma<\infty)$ be the resolution of the identity for the operator $L_{1}$. Suppose that $[a, b]$ is as given above. Then for any $f$ in $\mathscr{H}_{1}$ with bounded support $E_{1}(\sigma) f, \sigma \in[a, b]$, is absolutely continuous with respect to the Lebesgue measure:

$$
\frac{d}{d \sigma}\left(E_{1}(\sigma) f\right)=\frac{1}{2 \pi i}\left\{v_{+}(\cdot, \sigma)-v_{-}(\cdot, \sigma)\right\} \quad \text { in } \mathcal{K}_{1+\delta}
$$

Proof. (4.21) follows from the integral representation of the resolvent and the Stieltjes inversion formula since $v_{\varepsilon}(\cdot, \sigma)$ $=\left(L_{1}-\sigma-i \varepsilon\right)^{-1} f$ converges as $\varepsilon \rightarrow \pm 0$ to $v_{ \pm}(\cdot, \sigma)$ uniformly with respect to $\sigma \in[a, b]$.

q. e. d.

Finally we remark that the following theorem can be proved by the same line of proof given by Éidus [2; Theorem 5] (cf., also Remark 4.3):

Theorem 4. 3. Suppose that the operator $L$ is elliptic and inequality (2.10) holds for any $f$ in $\mathscr{D}\left(L_{1}\right)$. Then $v_{+}(\cdot, \sigma)$ are $\mathcal{K}_{3+\delta^{-}}$ valued Hölder continuous functions of $\sigma \in[a, b]$, where $[a, b]$ is any interval as given above, i.e., there exist positive constants $C$ and $h^{\prime}(<h<1)$ such that

$$
\left|v_{+}(\cdot, \mu)-v_{+}(\cdot, \sigma)\right|_{3+\delta} \leq C|\mu-\sigma|^{h^{\prime}} \quad \text { for any } \mu, \sigma \text { in }[a, b] \text {. }
$$

\section{Eigenfunction Expansions}

The main results of this $\S$ and the next $\S 6$ can be treated in the framework of the theory developed by many authors for the Schrödinger and the wave equations in an exterior domain (cf., Shizuta [21], Ikebe [5, 6], Shenk [20], and Lax and Phillips [12, 13]).

\section{1. Distorted plane waves.}

We first establish the existence and properties of distorted waves $\left\{V_{\nu}(x, \xi ; \lambda) ; \nu=1,2, \cdots, k\right\}$ defined for $x \in \bar{G}, \xi \in \boldsymbol{R}^{n}$ and $\operatorname{Im} \lambda \geq 0$ (or $\operatorname{Im} \lambda \leq 0)$. These results will enable us to show that the distorted plane waves 


$$
\begin{gathered}
\Phi_{\nu}^{ \pm}(x, \xi)=P_{\nu}(\xi) e^{i x \cdot \xi}+V_{\nu}^{ \pm}(x, \xi) \quad(\nu \neq(k+1) / 2) ; \\
V_{\nu}^{ \pm}(x, \xi)=V_{\nu}\left(x, \xi ; \tau_{\nu}|\xi| \mp i 0\right),
\end{gathered}
$$

are a complete set of generalized eigenfunctions for $L_{1}$.

Let $\alpha(x)$ be a $C^{\infty}$ scalar function such that

$$
\alpha(x)=\left\{\begin{array}{lll}
1 & \text { for } & |x|<\rho \\
0 & \text { for } & |x|>\rho+1
\end{array}\right.
$$

Consider the following equation in $\mathscr{H}_{1}$ :

$$
\begin{gathered}
\left(L_{1}-\lambda\right) \Psi_{\nu}=-\left(L_{1}-\tau_{\nu}|\xi|\right)(1-\alpha) \Phi_{\nu}^{0}+\left(\tau_{\nu}|\xi|-\lambda\right) \alpha M^{-1 / 2} \Phi_{\nu}^{0} \\
(\nu=1,2, \cdots, k)
\end{gathered}
$$

where we put

$$
\Phi_{\nu}^{0}(x, \xi)=P_{\nu}(\xi) e^{i x \cdot \xi},
$$

and the matrix valued function $\Psi_{\nu}$ is said to be in $\mathscr{H}_{1}$ if its each column or row vector is in $\mathscr{H}_{1}$. Since $L_{1}$ equals $L_{0}$ for $|x|>\rho$ and $\left(L_{0}-\tau_{\nu}|\xi|\right) \Phi_{\nu}^{0}=0$, it follows that the right side of this equation has its support in the ball $\{|x|<\rho+1\}$. Thus, for each $\lambda$ non-real there exists a unique solution $\Psi_{\nu}=\Psi_{\nu}(\cdot, \xi ; \lambda) \in \mathscr{D}\left(L_{1}\right)$ of this equation. Further, according to Theorem 4.2, the limits

$$
\Psi_{\nu}(x, \xi ; \sigma \mp i 0)=\lim _{\varepsilon \rightarrow \mp 0} \Psi_{\nu}(x, \xi ; \sigma+i \varepsilon) \equiv \Psi_{\nu}^{ \pm}(x, \xi)
$$

exist if $\sigma$ is not in $\sigma_{p}\left(L_{1}\right) \cup\{0\}$, and satisfy the incoming (-) and outgoing $(+)$ radiation conditions, respectively.

We put

$$
V_{\nu}(x, \xi ; \lambda)=\Psi_{\nu}(x, \xi ; \lambda)-\alpha(x) \Phi_{\nu}^{0}(x, \xi) .
$$

Then we can summarize the properties of $V_{\nu}$ as follows:

Lemma 5. 1. For each $\lambda$ non-real $V_{\nu}(\cdot, \xi ; \lambda)(\nu=1,2, \cdots, k)$ are $\mathscr{H}_{1}$-valued bounded, continuous functions of $\xi \in \boldsymbol{R}^{n}-\{0\}$. Let $[a, b]$ be any interval in $\boldsymbol{R}^{1}-\left[\sigma_{p}\left(L_{1}\right) \cup\{0\}\right]$. Then $V_{\nu}(\cdot, \xi ; \sigma \pm i \varepsilon)$ are $\mathcal{K}_{1+\delta^{-}}$ valued bounded, continuous functions of $\xi \in \boldsymbol{R}^{n}-\{0\}, \sigma \in[a, b]$ and $\varepsilon \in[0,1)$. Further $V_{\nu}^{-}=V_{\nu}(x, \xi ; \sigma+i 0)\left(V_{\nu}^{+}=V_{\nu}(x, \xi ; \sigma-i 0)\right)$ satisfies the incoming (outgoing) radiation condition.

Proof. The assertion is already proved for $\Psi_{\nu}(\cdot, \xi ; \sigma+i \varepsilon)$. 
Since $\alpha$ has its support in $|x|<\rho+1$ and $\Phi_{\nu}^{0}(x, \xi)$ are bounded, continuous in $x \in \boldsymbol{R}^{n}$ and $\xi \in \boldsymbol{R}^{n}-\{0\}$, we conclude the lemma noting (5.6).

q. e.d.

We put

$$
\begin{aligned}
\Phi_{\nu}(x, \xi ; \lambda) & =\Phi_{\nu}^{0}(x, \xi)+V_{\nu}(x, \xi ; \lambda) \\
& =\{1-\alpha(x)\} \Phi_{\nu}^{0}(x, \xi)+\Psi_{\nu}(x, \xi ; \lambda) .
\end{aligned}
$$

Then, since $(1-\alpha) \Phi_{\nu}^{0}$ satisfies the boundary condition (2.3), we have easily from equation (5.4) that $\Phi_{\nu}(\cdot, \xi ; \lambda) \in \mathscr{D}_{\text {loc }}\left(L_{1}\right)$ and

$$
\left(L_{1, l o c}-\lambda\right) \Phi_{\nu}=\left(\tau_{\nu}|\xi|-\lambda\right) M^{-1 / 2} \Phi_{\nu}^{0}
$$

taking into account that $M(x)=I$ for $|x|>\rho$. Finally note that the distorted plane waves $\Phi_{\nu}^{ \pm}(x, \xi)(\nu \neq(k+1) / 2)$ can be defined by (5.1) for any $\xi \in \boldsymbol{R}^{n}$ such that $\tau_{\nu}|\xi|$ is not in $\sigma_{p}\left(L_{1}\right) \cup\{0\}$ and satisfy the equations

$$
\left(I_{1, \text { loc }}-\tau_{\nu} \mid \xi_{\mid}\right) \Phi_{\nu}^{ \pm}=0 .
$$

\section{2. Generalized Fourier inversion formula; the expansion} theorem.

Let $f(x)$ be any function in $\left[C_{0}^{\infty}\left(\boldsymbol{R}^{n}\right)\right]^{m}$ and let $\lambda$ be non-real. Define the following transforms of $f$ :

$$
W_{\nu}(x, \lambda ; f)=(2 \pi)^{-n / 2} \int_{\boldsymbol{R}^{n}} \frac{\Phi_{\nu}(x, \xi ; \lambda) \hat{f}(\xi)}{\tau_{\nu}|\xi|-\lambda} d \xi \quad(\nu=1,2, \cdots, k) .
$$

Because of the summability of $\hat{f}(\xi)$ and (5.8) we have $W_{\nu} \in \mathscr{D}_{l o c}\left(L_{1}\right)$ and

$$
\begin{aligned}
& \left(L_{1, l o c}-\lambda\right) W_{\nu}(x, \lambda ; f)=M^{-1 / 2}(x) f_{\nu}(x) ; \\
& f_{\nu}(x)=(2 \pi)^{-n / 2} \int_{\boldsymbol{R}^{n}} e^{i x \cdot \xi} P_{\nu}(\xi) \hat{f}(\xi) d \xi .
\end{aligned}
$$

On the other hand, since

$$
W_{\nu} \equiv\left(L_{0}-\lambda\right)^{-1} f_{\nu}+(2 \pi)^{-n / 2} \int_{\boldsymbol{R}^{n}} \frac{V_{\nu}(\cdot, \xi ; \lambda) \hat{f}(\xi)}{\tau_{\nu}|\xi|-\lambda} d \xi,
$$

it follows that $W_{\nu} \in \mathscr{H}_{1}$ if we note that $V_{\nu} \in \mathscr{H}_{1}$ for $\lambda$ non-real. Thus, noting that $\sum_{\nu=1}^{k} f_{\nu}(x)=f(x)$, we have from (5.11) 


$$
\left(L_{1}-\lambda\right)^{-1} M^{-1 / 2} f=\sum_{\nu=1}^{k}\left(L_{1}-\lambda\right)^{-1} M^{-1 / 2} f_{\nu}=\sum_{\nu=1}^{k} W_{\nu}(\cdot, \lambda ; f) .
$$

For $f$ in $\left[C_{0}^{\infty}(\Lambda)\right]^{m}(\Lambda$ the interior of $\partial G)$, they imply that

$$
\sum_{\nu=1}^{k} W_{\nu}(x, \lambda ; f)=0 \quad \text { for } x \text { in } G
$$

since $\lambda$ non-real cannot be an eigenvalue of $L_{1}$.

Now, put $R_{1}(\lambda)=\left(L_{1}-\lambda\right)^{-1}$ ( $\lambda$ non-real). It follows from the resolvent equation that

$$
\begin{aligned}
& \left(\left\{R_{1}(\sigma+i \varepsilon)-R_{1}(\sigma-i \varepsilon)\right\} f, g\right)_{1}=2 i \varepsilon\left(R_{1}(\sigma \pm i \varepsilon) f, R_{1}(\sigma \pm i \varepsilon) g\right)_{1} \\
& \quad=2 i \varepsilon \int_{\boldsymbol{R}^{n}} M^{1 / 2}(x)\left[R_{1}(\sigma \pm i \varepsilon) f\right](x) \cdot \overline{M^{1 / 2}(x)\left[R_{1}(\sigma \pm i \varepsilon) g\right](x)} d x
\end{aligned}
$$

for $f, g$ in $\mathscr{H}_{1}$, where $(\cdot, \cdot)_{1}$ denotes the innerproduct in $\mathscr{H}_{1}$ and the function $M^{1 / 2} R_{1}(\sigma \pm i \varepsilon) f$ and $M^{1 / 2} R_{1}(\sigma \pm i \varepsilon) g$ have been extended to the interior domain $\Lambda$ to be zero. We consider the Fourier transforms of these functions. We have for $h, f$ in $\left[C_{0}^{\infty}\left(\boldsymbol{R}^{n}\right)\right]^{m}$

$$
\begin{gathered}
\left(W_{\nu}(\cdot, \lambda ; h), f\right)_{1}=(2 n)^{-n / 2} \int_{G} M(x) \int_{\boldsymbol{R}^{n}} \frac{\Phi_{\nu}(x, \xi ; \lambda) \hat{h}(\xi)}{\tau_{\nu}|\xi|-\lambda} d \xi \cdot \overline{f(x)} d x \\
=(2 n)^{-n / 2} \int_{\boldsymbol{R}^{n}} \frac{\hat{h}(\xi)}{\tau_{\nu}|\xi|-\lambda} \cdot \int_{G} \overline{\Phi_{\nu}^{*}(x, \xi ; \lambda) M(x) f(x)} d x d \xi .
\end{gathered}
$$

Since $\left(W_{\nu}(\cdot, \lambda ; h), f\right)_{1}=\left(h,\left[M^{1 / 2} R_{1}(\bar{\lambda}) f\right]_{\nu}\right)^{10)}$ by $(5.12)$, it follows that (5.15) $P_{\nu}(\xi)\left[M^{1 / 2} R_{1}(\bar{\lambda}) f\right]^{\wedge}(\xi)=\frac{(2 \pi)^{-n / 2}}{\tau_{\nu}|\xi|-\lambda} \int_{G} \Phi_{\nu}^{*}(x, \xi ; \lambda) M(x) f(x) d x$.

We define the transform $\hat{f}_{2}(\xi, \lambda)$ of functions $f$ in $\left[C_{0}^{\infty}(G)\right]^{m}$ by

$$
\hat{f}_{\nu}(\xi, \lambda)=(2 \pi)^{-2 / n} \int_{G} \Phi_{\nu}^{*}(x, \xi ; \bar{\lambda}) M(x) f(x) d x .
$$

Then, from (5.14) and (5.15), we have using the Parseval equality

$$
\begin{aligned}
& \left(\left\{R_{1}(\sigma+i \varepsilon)-R_{1}(\sigma-i \varepsilon)\right\} f, g\right)_{1} \\
& \quad=\sum_{\nu=1}^{k} \int_{\mathbb{R}^{n}} \frac{2 i \varepsilon}{\left(\tau_{\nu}|\xi|-\sigma\right)^{2}+\varepsilon^{2}} \hat{f}_{\nu}(\xi, \sigma \mp i \varepsilon) \cdot \overline{\hat{g}_{\nu}(\xi, \sigma \mp i \varepsilon)} d \xi
\end{aligned}
$$

for $f, g$ in $\left[C_{0}^{\infty}(G)\right]^{m}$.

10) $(f, g)=\int_{\theta} f(x) \cdot \overline{g(x)} x d$. 
Let $E_{1}(\sigma)(-\infty<\sigma<\infty)$ denote the resolution of the identity for $L_{1}$. For any interval $[a, b]$ in $\boldsymbol{R}^{1}-\left[\sigma_{p}\left(L_{1}\right) \cup\{0\}\right]$ we have

(5.18) $\left(\left\{E_{1}(b)-E_{1}(a)\right\} f, g\right)_{1}=\frac{1}{2 \pi i} \lim _{\varepsilon \rightarrow+0} \int_{a}^{b}\left(\left\{R_{1}(\sigma+i \varepsilon)-R_{1}(\sigma-i \varepsilon)\right\} f, g\right)_{1} d \sigma$.

Hence, putting

$$
\hat{f}_{\nu}^{ \pm}(\xi)=\hat{f}_{\nu}\left(\xi, \tau_{\nu}|\xi| \mp i 0\right)
$$

we have easily from (5.17) and (5.18)

$$
\begin{aligned}
& \left(\left\{E_{1}(b)-E_{1}(a)\right\} f, g\right)_{1}= \\
& \quad=\left\{\begin{array}{lll}
\sum_{\nu=1}^{[k / 21} \int_{K_{\nu}(a, b)} \hat{f}_{\nu}^{ \pm}(\xi) \cdot \overline{\hat{g}_{\nu}^{ \pm}(\xi)} d \xi \quad \text { if } & 0<a<b \\
\sum_{\nu=1}^{[k / 2 / 2]} \int_{K_{\nu}(a, b)} \hat{f}_{k-\nu+1}^{ \pm}(\xi) \cdot \overline{\hat{g}_{k-\nu+1}^{ \pm}(\xi)} d \xi & \text { if } \quad 0>b>a,
\end{array}\right.
\end{aligned}
$$

where $K_{\nu}(a, b)=\left\{\xi ; a<\tau_{\nu}|\xi|<b\right\} \quad$ if $\quad 0<a<b$, and $=\{\xi ; a<$ $\left.-\tau_{\nu}|\xi|<b\right\}$ if $0>b>a$. These imply that for $f$ in $\left[C_{0}^{\infty}(G)\right]^{m}$, each $\hat{f}_{\nu}^{ \pm}(\xi)$ is in $\left[L^{2}\left(K_{\nu}(a, b)\right)\right]^{m}$ and can be extended by continuity to an isometry of $\left\{E_{1}(b)-E_{1}(a)\right\} \mathscr{H}_{1}$ into $P_{\nu}(\xi)\left[L^{2}\left(K_{\nu}(a, b)\right)\right]^{m}$. Further, if we note that $\sigma_{p}\left(L_{1}\right)$ forms a discrete set in $\boldsymbol{R}^{1}$, then it follows easily from (5.20) that

$$
(f, g)_{1}=\sum_{\nu=1}^{[k / 2]} \int_{\boldsymbol{R}^{n}}\left\{\hat{f}_{\nu}^{ \pm}(\xi) \cdot \overline{\hat{g}_{\nu}^{ \pm}(\xi)}+\hat{f}_{k-\nu+1}^{ \pm}(\xi) \cdot \overline{\hat{g}_{k-\nu+1}^{ \pm}(\xi)}\right\} d \xi
$$

for $f, g$ in $(I-Q) \mathcal{H}_{1}$, where $Q$ denotes the projection onto the eigenspace of $L_{1}$ corresponding to the all eigenvalues.

Summing up we can now state the following expansion theorem.

Theorem 5.1. The distorted plane waves $\left\{\Phi_{\nu}^{ \pm}(\cdot, \xi), \xi \in \boldsymbol{R}^{n}\right.$ $-\{0\}, \nu \neq(k+1) / 2\}$ form a complete set of generalized eigenfunctions of $L_{1}$ restricted to $(I-Q) \mathscr{A}_{1}$ :

(1) Put $Z^{ \pm}=\sum_{\nu=1}^{[k / 2]}\left(Z_{\nu}^{ \pm}+Z_{k-\nu+1}^{ \pm}\right)$, where

$$
\left[Z_{\nu}^{ \pm} f\right]^{\wedge}(\xi)=(2 \pi)^{-n / 2} \int_{G} \Phi_{\nu}^{ \pm *}(x, \xi) M(x) f(x) d x, \quad \nu \neq(k+1) / 2 .
$$

Then $Z^{ \pm}$is an isometry of $(I-Q) \mathscr{H}_{1}$ onto $\mathscr{M}_{ \pm} \subset\left(I-Q_{0}\right) \mathscr{H}_{0}=\mathscr{H}_{0} \ominus$ $\Re\left(L_{0}\right)$. The adjoint operator $W^{ \pm}$of $Z^{ \pm}$is an isometry of $\mathscr{M}_{ \pm}$onto 
an $(I-Q) \mathcal{H}_{1}$ and is given by $W^{ \pm}=\sum_{\nu=1}^{[k / 2]}\left(W_{\nu}^{ \pm}+W_{k-\nu+1}^{ \pm}\right)$, where

$$
\left[W_{\nu}^{ \pm} f\right](x)=(2 \pi)^{-n / 2} \int_{\mathbb{R}^{n}} \Phi_{\nu}^{ \pm}(x, \xi) \hat{f}(\xi) d \xi, \quad \nu \neq(k+1) / 2 .
$$

Here we take the integrals (5.22) and (5.23) in the sense of the limit in the mean.

(2) The expansion formula of an arbitrary function $f$ in $\mathcal{H}_{1}$ can be obtained as follows:

$$
\begin{aligned}
& {[(I-Q) f](x)=\left[W^{ \pm} Z^{ \pm} f\right](x)=} \\
& \quad=\sum_{\nu=1}^{[k / 21}(2 \pi)^{-n / 2} \int_{\mathbb{R}^{n}}\left\{\Phi_{\nu}^{ \pm}(x, \xi) \hat{f}_{\nu}^{ \pm}(\xi)+\Phi_{k-\nu+1}^{ \pm}(x, \xi) \hat{f}_{k-\nu+1}^{ \pm}(\xi)\right\} d \xi \\
& \quad=\sum_{\nu=1}^{[k / 2]}\left\{\left[W_{\nu}^{ \pm} Z_{\nu}^{ \pm} f\right](x)+\left[W_{k-\nu+1}^{ \pm} Z_{{ }_{k}-\nu+1}^{ \pm} f\right](x)\right\} .
\end{aligned}
$$

(3) $f \in(I-Q) \mathscr{D}\left(L_{1}\right)$ if and only if each $|\xi| \hat{f}_{\nu}^{ \pm}(\xi)(\nu \neq(k+1) / 2)$ is in $P_{\nu}(\xi) \hat{\mathscr{M}}_{0}$. We have the following diagonal representation of $L_{1}$ : (5. 25) $\left[(I-Q) L_{1} f\right](x)=$

$$
=\sum_{\nu=1}^{[k / 2]}(2 \pi)^{-n / 2} \int_{R^{n}} \tau_{\nu}|\xi|\left\{\Phi_{\nu}^{ \pm}(x, \xi) \hat{f}_{\nu}^{ \pm}(\xi)-\Phi_{k-\nu+1}^{ \pm}(x, \xi) \hat{f}_{k-\nu+1}^{ \pm}(\xi)\right\} d \xi .
$$

Proof. We have already proved assertions (1) and (2). Assertion (3) follows easily from the relation

$$
\begin{aligned}
{\left[L_{1} f\right]_{\nu}^{\wedge \pm}(\xi) } & =(2 \pi)^{-n / 2} \int_{G}\left[L_{1, l o c} \Phi_{\nu}^{ \pm}(x, \xi)\right]^{*} M(x) f(x) d x \\
& =(2 \pi)^{-n / 2} \tau_{\nu}|\xi| \int_{G} \Phi_{\nu}^{ \pm *}(x, \xi) M(x) f(x) d x,
\end{aligned}
$$

which is easily verified by use of (5.9), and the fact that $\tau_{k-\nu+1}=-\tau_{\nu}$. q.e.d.

\section{3. Orthogonality of the eigenfunctions.}

Theorem 5.2. The range $\mathscr{M}_{ \pm}$of each $Z^{ \pm}$is equal to $\left(I-Q_{0}\right) \mathscr{H}_{0}$. Namely the following formula holds for any $f(x)$ in $\mathcal{H}_{0}$.

$$
\left[\left(I-Q_{0}\right) f\right](x)=\left[Z^{ \pm} W^{ \pm} f\right](x) .
$$

Proof. We have only to show that $P_{\nu}(\xi) \hat{f}(\xi)=\left[Z_{\nu}^{ \pm} W_{\nu}^{ \pm} f\right]^{\wedge}(\xi)$. This can be proved by the same argument as given by Ikebe [5]. Put 


$$
\begin{aligned}
& \hat{g}(\xi)=P_{\nu}(\xi) \hat{f}(\xi)-\left[Z_{\nu}^{+} W_{\nu}^{+} f\right]^{\wedge}(\xi), \\
& s_{\rho}(x)=(2 \pi)^{-n / 2} \int_{|\xi| \leq \rho} \Phi_{\nu}^{+}(x, \xi) \hat{g}(\xi) d \xi, \\
& S_{\rho}(x, \lambda)=\left(L_{1}-\lambda\right)^{-1} s_{\rho}=\int_{|\xi| \leq \rho} \frac{\Phi_{\nu}^{+}(x, \xi) \hat{g}(\xi)}{\tau_{\nu}|\xi|-\lambda} d \xi,
\end{aligned}
$$

where $\lambda=\sigma+i \varepsilon, \varepsilon>0$. Since $s_{\rho} \rightarrow W_{\nu}^{+} g=W_{\nu}^{+} f-W_{\nu}^{+} Z_{\nu}^{+} W_{\nu}^{+} f=0$ in $\mathscr{H}_{1}$ as $\rho \rightarrow \infty$, we have easily

$$
\begin{gathered}
0=\lim _{\varepsilon \rightarrow+0}(2 \pi)^{-n / 2} \int_{a}^{b} d \sigma \int_{\boldsymbol{R}^{n}} \frac{2 i \varepsilon \hat{g}(\xi) d \xi}{\left(\tau_{\nu}|\xi|-\lambda\right)^{2}+\varepsilon^{2}} \int_{D} \Phi_{\nu}^{+}(x, \xi) d x \\
=(2 \pi)^{-n / 2} 2 \pi i \int_{D} d x \int_{K_{\nu}(a, b)} \Phi_{\nu}^{+}(x, \xi) \hat{g}(\xi) d \xi,
\end{gathered}
$$

where $D$ is an arbitrary bounded domain in $G$, and $[a, b]$ is an arbitrary interval in $(0, \infty)$. Thus we get

$$
\int_{\tau_{\nu}|\xi|=r} \Phi_{\nu}^{+}(x, \xi) \hat{g}(\xi) d S_{\xi}=0
$$

for almost every positive $r$. Define the function

$$
u(x, r)=\int_{\tau_{\nu}|\xi|=r} e^{i x \cdot \xi} P_{\nu}(\xi) \hat{g}(\xi) d S_{\xi}=\int_{\tau_{\nu}|\xi|=r}-V_{\nu}^{+}(x, \xi) \hat{g}(\xi) d S_{\xi} .
$$

Then $u(x, r)$ is everywhere defined in $\boldsymbol{R}^{n}$ and satisfies $\left(L_{0}-\tau_{\nu}|\xi|\right) u$ $=0$. On the other hand, the third member shows that $u$ satisfies the outgoing radiation condition (Lemma 5.1). Hence, by Lemma 4. 2, $u$ must be in $\mathcal{H}_{0}$. However, since $L_{0}$ has no eigenvalue except the origin 0 , we conclude that $u(x, r)$ must vanish identically, and hence that $g(x)=\int_{0}^{\infty} u(x, r) d r=0$.

q. e. d.

The formulae (5.34) and (5.26) imply the unitary equivalence between $L_{0}$ and $L_{1}$ :

Corollary 5.1. $(I-Q) L_{1}$ is unitarily equivalent to $\left(I-Q_{0}\right) L_{0}$ :

$$
(I-Q) L_{1}=W^{ \pm}\left(I-Q_{0}\right) L_{0} Z^{ \pm}, \quad \text { where } Z^{ \pm}=W^{ \pm *}=W^{ \pm-1} \text {. }
$$

\section{The Wave and Scattering Operators}

For $-\infty<t<\infty$ we define the operator $W(t): \mathscr{H}_{0} \rightarrow \mathcal{H}_{1}$ by

$$
W(t)=e^{i L_{1} t} J e^{-i L_{0} t}\left(I-Q_{0}\right),
$$


where $J: \mathscr{H}_{0} \rightarrow \mathscr{H}_{1}$ is the truncation operator defined by

$$
[J f](x)=f(x) \quad(x \in G) \text {. }
$$

The strong limits as $t \rightarrow \pm \infty$ of $W(t)$ are, if they exist, called the wave operators. Our first aim in this $\S$ is to show that the wave operators exist and are equal to $W^{ \pm}$given in the preceding section.

Lemma 6.1. As $t \rightarrow \pm \infty, e^{-i L_{0} t}\left(I-Q_{0}\right)$ converges to zero in the sense of local energy norm: Let $G^{\prime}$ be any bounded domain in $\boldsymbol{R}^{n}$. Then

$$
\lim _{t \rightarrow \pm \infty}\left\|e^{-i L_{0} t}\left(I-Q_{0}\right) f\right\|_{0, G^{\prime}}=0 \quad \text { for any } f \text { in } \mathcal{H}_{0} \text {. }
$$

Proof. The lemma is easily proved by use of the Fourier inversion formula and the Riemann-Lebesgue theorem (cf. Ikebe [6]).

q. e. d.

The following result is due to Wilcox ([22]; Theorem 6.1).

Lemma 6. 2. ${ }^{11)}$ Let $\gamma(x)$ be a Hermitian matrix valued function which is bounded measurable in $x$ and vanishes for sufficiently large $|x|$. Then for any $g$ in $\left[C_{0}^{\infty}\left(\boldsymbol{R}^{n}\right)\right]^{m},\left\|\gamma L_{0} e^{ \pm i L_{0} t} g\right\|_{0}$ is integrable in $t_{0}<t<\infty\left(t_{0}>0\right)$.

Using the above two lemmas, we can prove the following theorem :

Theorem 6.1. $W^{ \pm}$coincide with the wave operators for the pair $L_{0}, L_{1}$ in the time dependent formulation, i.e., we have

$$
W^{ \pm}=s-\lim _{t \rightarrow \pm \infty} W(t)
$$

Proof. We set

$$
\begin{aligned}
W(t) & =e^{i L_{0} t}\left(1-\beta_{r}\right) J e^{-i L_{0} t}\left(I-Q_{0}\right)+ \\
& +e^{i L_{1} t} \beta_{r} J e^{-i L_{0} t}\left(I-Q_{0}\right) \equiv W_{0}(t)+\tilde{W}(t),
\end{aligned}
$$

where $\beta_{r}=\beta_{r}(|x|)(x \in G)$ is the real valued scalar function given by (4.7). Since $e^{i L_{1} t}$ is unitary in $\mathscr{H}_{1}$, we have for any $f$ in $\mathscr{H}_{0}$

11) In Theorem 6.1 of [22] it is not assumed that $L_{0}$ is isotropic and $r$ has compact support. It is sufficient to assume that $L_{0}$ is uniformly propagative and $\gamma(x)$ behaves like $0\left(|x|^{-p}\right)(p>1)$ at infinity. 
(6.6)

$$
\begin{aligned}
& \left\|W_{0}(t) f\right\|_{1} \leq\left\|\left(1-\beta_{r}\right) J e^{-i L_{0} t}\left(I-Q_{0}\right) f\right\|_{1} \\
& \quad \leq \text { const } \| e^{-i L_{0} t\left(I-Q_{0}\right) f \|_{0, G_{r+1}} \rightarrow 0 \quad(\text { as } t \rightarrow \pm \infty)}
\end{aligned}
$$

by Lemma 6.1. Thus our problem is reduced to showing that

(6. 7) $I-Q_{0}=s-\lim _{t \rightarrow \pm \infty} Z^{ \pm} \widetilde{W}(t)=s-\lim _{t \rightarrow \pm \infty} e^{i L_{0} t} Z^{ \pm} \beta_{r} J e^{-i L_{0} t}\left(I-Q_{0}\right)$,

where we have used the formulae (5.26) and

$$
Z^{ \pm} e^{i L_{1} t}=e^{i L_{0} t} Z^{ \pm}
$$

which easily follows from the fact that the unitary equivalence between $L_{0}\left(I-Q_{0}\right)$ and $L_{1}(I-Q)$ is established by $Z^{ \pm}=W^{ \pm *}$. Since $Z_{\nu}^{+}$can be represented in the Fourier spaces as $\left[Z_{\nu}^{+} g\right]^{\wedge}(\xi)=\hat{g}_{\nu}^{+}(\xi) \in$ $P_{\nu}(\xi) \hat{\mathcal{H}}_{0}$, it is sufficient to show that

(6. 8) $f_{\nu} \equiv(2 \pi)^{-n / 2} \int_{\boldsymbol{R}^{n}} \Phi_{\nu}^{0}(\cdot, \xi) \hat{f}(\xi) d \xi=s-\lim _{t \rightarrow \infty} e^{i L_{0} t} Z_{\nu}^{+} \beta_{r} J e^{-i L_{0} t}\left(I-Q_{0}\right) f$

$$
=s-\lim _{t \rightarrow \infty}(2 \pi)^{-n / 2} \int_{\boldsymbol{R}^{n}} \Phi_{\nu}^{0}(\cdot, \xi) e^{i \tau_{\nu}|\xi| t}\left[\beta_{r} J e^{-i L_{0} t}\left(I-Q_{0}\right) f\right]_{\nu}^{\wedge+}(\xi) d \xi
$$

for any $f$ in $\mathscr{H}_{0}$ and $\nu \neq(k+1) / 2$. Set $h=\left(I-Q_{0}\right) f$. Choose $r>\rho+1$. It follows from (5.1) that

$$
\begin{aligned}
& {\left[\beta_{r} J e^{-i L_{0} t} h\right]_{\nu}^{\Lambda+}(\xi) } \\
&=(2 \pi)^{-n / 2} \int_{G} \Phi_{\nu}^{+*}(x, \xi) \beta_{r}(|x|)\left[e^{-i L_{0} t} h\right](x) d x \\
&=(2 \pi)^{-n / 2} \int_{\boldsymbol{R}^{n}} \Phi_{\nu}^{0 *}(x, \xi)\left[e^{-i L_{0} t} h\right](x) d x \\
&-(2 \pi)^{-n / 2} \int_{\boldsymbol{R}^{n}} \Phi_{\nu}^{0 *}(x, \xi)\left\{1-\beta_{r}(|x|)\right\}\left[e^{-i L_{0} t} h\right](x) d x \\
&+(2 \pi)^{-n / 2} \int_{G} V_{\nu}^{+*}(x, \xi) \beta_{r}(|x|)\left[e^{-i L_{0} t} h\right](x) d x \\
& \equiv \hat{U}_{\nu, 1}(\xi, t)+\hat{U}_{\nu, 2}(\xi, t)+\hat{U}_{\nu, 3}(\xi, t) .
\end{aligned}
$$

where we have used the fact that $M(x)=I$ for $|x|>\rho$. Since $\hat{U}_{\nu, 1}(\xi, t)=P_{\nu}(\xi) e^{-i \tau_{\nu}|\xi| t} \hat{h}(\xi)$, we have

$$
\begin{aligned}
& (2 \pi)^{-n / 2} \int_{\boldsymbol{R}^{n}} \Phi_{\nu}^{0} \cdot(\cdot, \xi) e^{i \tau_{\nu}|\xi| t} \hat{U}_{\nu, 1}(\xi, t) d \xi \\
& =(2 \pi)^{-n / 2} \int_{\boldsymbol{R}^{n}} \Phi_{\nu}^{0}(\cdot, \xi) P_{\nu}(\xi) \hat{h}(\xi) d \xi \equiv h_{\nu}=\left[\left(I-Q_{0}\right) f\right]_{\nu}=f_{\nu} .
\end{aligned}
$$

On the other hand, we have 
(6. 10)

$$
\left\|e^{i L_{0} t} U_{\nu, 2}\right\|_{0}=\left\|U_{v, 2}\right\|_{0} \leq\left\|\left(1-\beta_{r}\right) e^{-i L_{0} t} h\right\|_{0} \rightarrow 0 \quad(t \rightarrow \infty)
$$

by the Parseval equality and Lemma 6.1. It remains to prove that

$$
\left\|e^{i L_{0} t} U_{\nu, 3}\right\|_{0} \rightarrow 0 \quad \text { as } t \rightarrow \infty .
$$

From (5.6) we have

$$
\beta_{r}(|x|) V_{\nu}^{+}(x, \xi)=\beta_{r}(|x|) \Psi_{\nu}\left(x, \xi ; \tau_{\nu}|\xi|-i 0\right) \equiv \beta_{r}(|x|) \Psi_{\nu}^{+}(x, \xi)
$$

since $\alpha \beta_{r}=0$ (note that $\alpha(x)$ is defined by (5.3)). Substitute this in the defining equation of $\hat{U}_{\nu, 3}(\xi, t)$. Then it follows that

$$
\begin{aligned}
\hat{U}_{\nu, 3}(\xi, t) & =(2 \pi)^{-n / 2} \int_{G} \Psi_{\nu}^{+*}(x, \xi) \beta_{r}(|x|)\left[e^{-i L_{0} t} h\right](x) d x \\
& =\lim _{\varepsilon \rightarrow+0}(2 \pi)^{-n / 2} \int_{G} \Psi_{\nu}^{*}\left(x, \xi ; \tau_{\nu}|\xi|-i \varepsilon\right) \beta_{r}(|x|)\left[e^{-i L_{0} t} h\right](x) d x .
\end{aligned}
$$

Since the functions in the right member of (5.4) have their supports in $\{|x|<\rho+1\}$, we have

$$
\begin{gathered}
\beta_{r}(|x|) \Psi_{\nu}(x, \xi ; \sigma-i \varepsilon)=\frac{1}{i}\left[\left(L_{0}-\sigma+i \varepsilon\right)^{-1} \gamma \Psi_{\nu}\right](x, \xi ; \sigma-i \varepsilon) ; \\
\gamma(x)=i\left[L_{0} \beta_{r}\right](x)=\beta_{r}^{\prime}(|x|) A^{0}\left(\frac{x}{|x|}\right)
\end{gathered}
$$

noting that $L_{1}=L_{0}$ for $|x|>r(>\rho)$. Thus

$$
\begin{aligned}
& U_{\nu, 3}(\xi, t) \\
& =\lim _{\varepsilon \rightarrow+0} i(2 \pi)^{-n / 2} \int_{G} \Psi_{\nu}^{*}\left(x, \xi ; \tau_{\nu}|\xi|-i \varepsilon\right) \gamma(x)\left(L_{0}-\tau_{\nu}|\xi|-i \varepsilon\right)^{-1}\left[e^{-i L_{0} t} h\right](x) d x \\
& =-\lim _{\varepsilon \rightarrow+0}(2 \pi)^{-n / 2} \int_{G} \Psi_{\nu}^{*}\left(x, \xi ; \tau_{\nu}|\xi|\right. \\
& \quad \times\left\{\int_{t}^{\infty} e^{i\left(\tau_{\nu}|\xi|+i \varepsilon\right)(s-t)}\left[e^{-i L_{0} s} h\right](x) d s\right\} d x .
\end{aligned}
$$

It is easy to see that

$$
\left\|e^{i L_{0} t} U_{\nu, 3}\right\|_{0} \leq \text { const }\|h\|_{0} \leq \text { const }\|f\|_{0} \quad\left(h=\left(I-Q_{0}\right) f\right) .
$$

Thus we have only to show (6.11) for $h$ in a dense domain of $\left(I-Q_{0}\right) \mathscr{H}_{0}$, that is, we can put $h=L_{0} g$, where $g$ is in $\left[C_{0}^{\infty}\left(\boldsymbol{R}^{n}\right)\right]^{m}$. Since, with $\gamma$ as in Lemma 6.2,

$$
\left\|\gamma e^{-i L_{0} t} h\right\|_{0}=\left\|\gamma L_{0} e^{-i L_{0} t} g\right\|_{0}
$$

is integrable in $t_{0}<t<\infty$ by Lemma 6 . 2, we finally obtain 


$$
\begin{aligned}
e^{i L_{0} t} U_{\nu, 3}(\cdot, t) & =(2 \pi)^{-n / 2} \int_{t}^{\infty} e^{i L_{0} s}\left[\gamma e^{-i L_{0} s} L_{0} g\right], d s \\
& -(2 \pi)^{-n / 2} \int_{t}^{\infty} e^{i L_{0} s} Z_{\nu}^{+} \gamma e^{-i L_{0} s} L_{0} g d s,
\end{aligned}
$$

where we have used the fact that $\Psi_{\nu}^{+}(x, \xi)=-\Phi_{\nu}^{0}(x, \xi)+\Phi_{\nu}^{+}(x, \xi)$ on the support $\{r<|x|<r+1\}$ of $\gamma(x)$. Hence we conclude that

$$
\left\|e^{i L_{0} t} U_{\nu, 3}\right\|_{0} \leq \mathrm{const} \int_{t}^{\infty}\left\|\gamma e^{-i L_{0} s} L_{0} g\right\|_{0} d s \rightarrow 0 \quad(\text { as } \quad t \rightarrow+\infty),
$$

proving (6.11).

Now the scattering operator $S$ acting in $\mathscr{H}_{0}$ is defined to be

$$
S=Z^{+} W^{-}=W^{+-1} W^{-} \text {. }
$$

Theorem 6.2. $S$ is unitary on $\left(I-Q_{0}\right) \mathscr{H}_{0}$ and is permutable with $L_{0}$. Further, for any function $f$ in $(I-Q) \mathcal{H}_{1}$ there exist two functions $f^{+}, f^{-}$in $\left(I-Q_{0}\right) H_{0}$ such that

$$
f^{+}=S f^{-},
$$

and

$$
\lim _{t \rightarrow \pm \infty}\left\|e^{-i L_{1} t} f-J e^{-i L_{0} t} f^{ \pm}\right\|_{1}=0 .
$$

Proof. The first assertion is obvious since $W^{-}:\left(I-Q_{0}\right) \mathscr{H}_{0} \rightarrow$ $(I-Q) \mathscr{H}_{1}, Z^{+}:(I-Q) \mathscr{H}_{1} \rightarrow\left(I-Q_{0}\right) \mathscr{H}_{0}$ are unitary, and since $L_{0} S$ $=Z^{+} W^{-} L_{0}=S L_{0}$ by (5.27). The second assertion is a direct consequence of Theorem 6.1 if we set $f^{ \pm}=Z^{ \pm} f$.

q. e. d.

\section{Examples}

\subsection{The Maxwell equation in an exterior domain.}

We consider the Maxwell system

$$
\left[\begin{array}{cc}
M_{1}(x) & 0 \\
0 & M_{2}(x)
\end{array}\right] \frac{\partial}{\partial t}\left[\begin{array}{l}
u_{1} \\
u_{2}
\end{array}\right]=\left[\begin{array}{cc}
0 & \operatorname{curl} \\
-\operatorname{curl} & 0
\end{array}\right]\left[\begin{array}{l}
u_{1} \\
u_{2}
\end{array}\right]
$$

in an exterior domain $G$ in $\boldsymbol{R}^{3}$, and we require the following boundary conditions :

(7.2) For each $z$ of the boundary $\partial G, u_{1}(z)+\alpha u_{2}(z)$ is parallel to the normal $n(z)$ to the boundary, where $\alpha$ is a given constant. 
We put

$$
M(x)=\left[\begin{array}{cc}
M_{1}(x) & 0 \\
0 & M_{2}(x)
\end{array}\right], \quad L=L_{0}=\frac{1}{i}\left[\begin{array}{cc}
0 & \text { curl } \\
-\operatorname{curl} & 0
\end{array}\right] .
$$

Here $M_{\nu}(x)(\nu=1,2)$ are $3 \times 3$ matrix valued functions satisfying the following

Conditions. (1) $M_{\nu}(x)$ are Hermitian, and postitive definite.

(2) $M_{\nu}(x)$ are $C^{2}$-class functions of $x$, and $M_{\nu}(x)=I$ for $|x|$ large.

(3) $M_{\nu}(x)$ curl-curl $M_{\nu}(x)$ define bounded operators in $\left[L^{2}(G)\right]^{3}$.

Remark. (3) is satisfied if $M_{\nu}(x)$ are diagonal : $M_{\nu}(x)=m_{\nu}(x) I$, where $m_{\nu}(x)$ are $C^{2}$-class scalar functions.

Under the above conditions on (7.4), we can verify that the system (7.1) with (7.2) satisfies all the assumptions (i vii) given in $\S 2$.

It is known by Schmidt ([18], Appendix 4 of [15]) that the boundary condition (7.2) satisfies assumptions (v) and (vi). Assumption (i $\sim \mathrm{iv}$ ) and (vii) are evident since we have

$$
\operatorname{det}\left[A^{0}(\xi)-\lambda\right]=\lambda^{2}(|\xi|-\lambda)^{2}(|\xi|+\lambda)^{2}
$$

\section{(cf., Courant and Hilbert [1]).}

Hence all of the preceding results can be applied to the system (7.1), (7.2). Namely, let $L_{0}$ be the Maxwell operator in the whole space $\boldsymbol{R}^{3}$ and $L$ be the same operator in the exterior domain $G \subset \boldsymbol{R}^{3}$ with the boundary condition (7.2), and put $L_{1}=M^{-1}(x) L$. Then $L_{0}$ and $L_{1}$ determine the selfadjoint operators in the Hilbert spaces $\mathscr{H}_{0}=\left[L^{2}\left(\boldsymbol{R}^{3}\right)\right]^{6}$ and $\mathscr{H}_{1}=\left[L^{2}(G)\right]^{6}$, respectively, where the norm of $\mathscr{H}_{1}$ is defined by

$$
\|f\|_{1}=\left\{\int_{G} M(x) f(x) \cdot \overline{f(x)} d x\right\}^{1 / 2} \quad\left(f=\mathscr{H}_{1}\right) .
$$

And we can prove the following theorem.

Theorem 7.1. The wave operators $W^{ \pm}$for the pair $L_{0}, L_{1}$ exist and are represented as

$$
\left[W^{ \pm} f\right](x)=(2 \pi)^{-3 / 2} \int_{\boldsymbol{R}^{n}}\left\{\Phi_{1}^{ \pm}(x, \xi)+\Phi_{3}^{ \pm}(x, \xi)\right\} \hat{f}(\xi) d \xi,
$$


where $\Phi_{\nu}^{ \pm}(x, \xi)(\nu=1,3)$ are the distorted planewaves solving the equation

$$
\left(L_{1, l o c}+(-1)^{(\nu+1) / 2}|\xi|\right) \Phi_{\nu}^{ \pm}(\cdot, \xi)=0 \quad(\nu=1,3) .
$$

$W^{ \pm}$are unitary operators from $\left(I-Q_{0}\right) \mathcal{H}_{0}$ onto $(I-Q) \mathcal{H}_{1}$, where $Q_{0}$ is the projection onto the null space $\Re\left(L_{0}\right)$ of $L_{0}$ and $Q$ is the projection onto the eigenspace of $L_{1}$ corresponding to the all eigenvalues of $L_{1}$ Hence the scattering operator $S$ defined to be

$$
S=Z^{+} W^{-}, \quad Z^{+}=W^{+*}=W^{+-1},
$$

becomes unitary operator on $\left(L-Q_{0}\right) \mathscr{H}_{0} . \quad$ It is obvious that

$$
Q_{0} \mathcal{H}_{0}=\mathscr{R}\left(L_{0}\right)=\left\{f_{2}(x)=(2 \pi)^{-3 / 2} \int_{\boldsymbol{R}^{n}} e^{i x \cdot \xi} P_{2}(\xi) \hat{f}(\xi) d \xi ; f \in \mathcal{H}_{0}\right\} .
$$

In the case that $M_{\nu}(x)=m_{\nu}(x) I$, we can say more:

Theorem 7.2. $L_{1}$ has no eigenvalue other than the origin 0 .

Proof. First note that

$$
\operatorname{div}\left[m_{1}(x) f_{1}(x)\right]=\operatorname{div}\left[m_{2}(x) f_{2}(x)\right]=0
$$

if $f=\left\{f_{1}, f_{2}\right\}$ belongs to $\mathscr{D}\left(L_{1}\right) \ominus \mathscr{N}\left(L_{1}\right){ }^{122}$ Suppose that $\sigma$ real $\neq 0$, and consider the equation

$$
\left(L_{1}-\sigma\right) \varphi=0 \quad\left(\varphi \in \mathcal{H}_{1}\right) .
$$

Put $M(x) \varphi=\psi=\left\{\psi_{1}, \psi_{2}\right\}$. Then it follows from (7.9) that

$$
\operatorname{div} \psi_{1}=\operatorname{div} \psi_{2}=0,
$$

and from (7.10) that

$$
L_{0} \psi-\sigma \psi=\tilde{N}(x, \sigma) \psi,
$$

where $N(\cdot, \sigma)$ is a matrix valued function having a bounded support. Applying $L_{0}+\sigma$ to (7.12) and noting (7.11), we finally get

$$
-\Delta \psi-\sigma^{2} \psi=\tilde{N}_{1}(x, \sigma) \psi \quad(x \in G),
$$

where $\tilde{N}_{1}(\cdot, \sigma)$ is also of bounded support. Since $\sigma^{2} \neq 0$ and $\psi \in \mathcal{H}_{1}$, this implies that $\psi=0$. q. e. d.

12) Cf. the lemma of Appendix 4 of [12]. 


\subsection{First-order systems in the whole space.}

We consider the system

$$
\frac{1}{i} M(x) \frac{\partial u}{\partial t}=L_{0} u=\frac{1}{i} \sum_{j=1}^{n} A_{j} \frac{\partial u}{\partial x_{j}}
$$

in the whole space $\boldsymbol{R}^{n}(n \geq 2)$. We assume that $M(x)$ satisfies assumptions (i), (iii) and (vii), and that $A^{\circ}(\xi)$ is isotropic (i. e., satisfies (iv)). Then it is obvious that $L_{0}$ in $\mathscr{H}_{0}=\left[L^{2}\left(\boldsymbol{R}^{n}\right)\right]^{m}$ and $L_{1}=M^{-1}(x) L_{0}$ in $\mathscr{H}_{1}=\left[L^{2}\left(\boldsymbol{R}^{n}\right)\right]^{m}$ are both selfadjoint, where the norm of $\mathscr{H}_{1}$ is defined by

$$
\|f\|_{1}=\left\{\int_{\boldsymbol{R}^{n}} M(x) f(x) \cdot \overline{f(x)} d x\right\}^{1 / 2} .
$$

Under the above assumptions (7.13) becomes a restricted case of uniformly propagative systems developed by Wilcox [22], Kato [10] and Ikebe [7]. They derived the existence and properties of the wave operators $W^{ \pm}$for the pair $L_{0}, L_{1}$. However, it remains unsolved whether $W^{ \pm}$are unitary.

We can solve this open problem, though our conditions are very strong compared with theirs, applying the preceding results to equation (7.14). For this aim we have only to show the following lemma :

Lemma 7.1. For any $0<r^{\prime}<r$, there exists a positive constant $C=C\left(r^{\prime}, r\right)$ such that

(7.14) $\sum_{j=1}^{n}\left\|\frac{\partial f}{\partial x_{j}}\right\|_{0, K_{r^{\prime}}} \leq C\left\{\|f\|_{0, K_{r}}+\left\|L_{0} f\right\|_{0, K_{r}}\right\}$ for any $f \in \mathscr{D}\left(L_{0}\right) \ominus \mathscr{N}\left(L_{0}\right)$.

\section{Proof. Since}

$$
\Re\left(L_{0}\right)=\left\{f_{(k+1) / 2}(x)=(2 \pi)^{-n / 2} \int_{\mathbb{R}^{n}} e^{i x \cdot \xi} P_{(k+1) / 2}(\xi) \hat{f}(\xi) d \xi ; f=\mathscr{H}_{0}\right\},
$$

we have only to show the inequality (7.14) for $f=g_{\nu}\left(g \in \mathscr{D}\left(L_{\nu_{0}}\right)\right)$, where

$$
g_{\nu}(x)=(2 \pi)^{-n / 2} \int_{\boldsymbol{R}^{n}} e^{i x_{*} \xi} P_{\nu}(\xi) \hat{g}(\xi) d \xi \quad(\nu \neq(k+1) / 2) .
$$

First we choose $g$ in $\left[C_{0}^{\infty}\left(\boldsymbol{R}^{n}\right)\right]^{m}$. Then, since $\left[L_{0} g_{v}\right]^{\wedge}(\xi)$ 
$=\tau_{\nu}|\xi| P_{\nu}(\xi) \hat{g}(\xi)$, it follows that $\left[L_{0}^{2} g_{\nu}\right](x)=-\tau_{\nu}^{2} \Delta g_{\nu}(x)$. Let $\alpha(x)$ be a $C^{\infty}$ scalar function such that $\alpha(x)=1$ for $x \in K_{r^{\prime}}$ and $=0$ for $x \in \mathbb{R}^{n}-K_{r}$. Then we have

$$
\sum_{j=1}^{n}\left\|\frac{\partial g_{\nu}}{\partial x_{j}}\right\|_{0, K_{r^{\prime}}} \leq \sum_{j=1}^{n}\left\|\frac{\partial \alpha g_{\nu}}{\partial x_{j}}\right\|_{0} \leq \text { const }\left\{\left\|g_{\nu}\right\|_{0, K_{r}}+|| L_{0} g_{\nu} \|_{0, K_{r}}\right\},
$$

where the "const" depends only on $\alpha(x)$ and $D_{j} \alpha(x)\left(D_{j}=\partial / \partial x_{j}\right.$; $j=1, \cdots, n)$. Since $\left[C_{0}^{\infty}\left(\boldsymbol{R}^{n}\right)\right]^{m}$ is dense in $\mathscr{D}\left(L_{0}\right)$, we get (7.14). q.e.d.

This lemma, with the assumptions on $M(x)$ and $A^{\prime}(\xi)$, shows that all the results in $\$ \$ 5,6$ can be applied to the operator $L_{1}$ corresponding to (7.13). Thus $W^{ \pm}$are shown to be unitary operators from $\left(I-Q_{0}\right) \mathcal{H}_{0}$ onto $(I-Q) \mathcal{H}_{1}$ by use of the completeness and the orthogonality of the generalized eigenfunctions (Theorems 5.1 and 5.2), and of an explicit construction for $W^{ \pm}$in terms of these expansions (Theorem 6.1).

\section{References}

[1] Courant, R., and D. Hilbert,, Mcthods of Mathematical Physics, II, Interscience, New York, 1962.

[2] Eidus, D. M., The principle of limiting absorption. Mat. Sb. (N. S.) 58 (100) (1962), 65-86 (Russian).

[3] Friedrichs, K. O., Ueber die Spectralzerlegung Integral-Operators, Mat. Ann. 115 (1938), 249-272.

[4] , Symmetric positive linear differential equations, Comm. Pure Appl. Math. 11 (1958), 333-410.

[5] Ikebe, T., Orthogonality of the eigen-function for the exterior problem connected with - $\Delta$, Arch. Rational Mech. Anal. 19 (1965), 71-73.

[6] - Scattering for the Schrödinger operator in an exterior domain, J. Math. Kyoto Univ. 7 (1967), 93-112.

[7] - Scattering for the uniformly propagative systems, to appear.

[8] Iwasaki, N., Local decay of solutions for symmetric hyperbolic systems with dissipative and coercive boundary conditions in exterior domains, Publ. RIMS Kyoto Univ., this issue.

[9] Kato, T., Perturbation Theory for Linear Operators, Springer, Berlin, 1966.

[10] - Scattering theory with two Hilbert spaces, J. Functional Anal. 1 (1967), 342-368.

「11] Lax, P. D., and R. S. Phillips, Local boundary conditions for dissipative symmetric linear differentisl operators, Comm. Purc Appl. Math. 13 (1960), 427-455.

[12] - Scattering Theory, Academic Press, New York, 1967.

[13] —, The acoustic equation with an indefinite energy form and the Schrodinger equation, J. Functional. Anal. 1 (1967), 37-83.

[14] Ludwig, D., The Radon transform on euclidean space, Comm. Pure Appl. Math. 19 (1966), 49-81. 
[15] Matsumura, M., Comportement des solutions de quelques problèmes mixtes pour certains systèmes hyperboliques symétriques à coefficients constants, Publ. RIMS Kyoto Univ. Ser. A 4 (1968), 309-359.

[16] - - Comportement asymptotique de solutions de certains problèmes mixtes pour des systèmes hyperboliques à coefficients constants, to appear.

[17] Mizohata, S., Theory of Partial Differential Equations, Iwanami, Tokyo, 1965 (Japanese).

[18] Mochizuki, K., Some remarks on radiation conditions, Proc. Japan Acad 45 (1969), to appear.

[19] Schmidt, G., Spectral and scattering theory for Maxwell's equations, Arch. Rational Mech. Anal. 28 (1968), 284-322.

[20] Shenk II, N. A., Eigenfunction expansions and scattering theory for the wave equation in an exterior region, Arch. Rational Mech. Anal. 21 (1966), 120-150.

[21] Shizuta, Y., Eigenfunction expansions associated with the operator $-\Delta$ in the exterior domain, Proc. Japan Acad. 39 (1963), 656-660.

[22] Wilcox, C. H., Wave operators and asymptotic solutions of wave propagation problems of classical physics, Arch. Rational Mech. Anal. 22 (1966), 37-78. 